\title{
LA-UR-14-27966
}

Approved for public release; distribution is unlimited.

Title: Neutron Propagation in Atmosphere

Author(s): $\quad$ Leacock, John

Intended for: Presentation

Issued: 
Disclaimer:

Los Alamos National Laboratory, an affirmative action/equal opportunity employer,is operated by the Los Alamos National Security, LLC for the National NuclearSecurity Administration of the U.S. Department of Energy under contract DE-AC52-06NA25396. By approving this article, the publisher recognizes that the U.S. Government retains nonexclusive, royalty-free license to publish or reproduce the published form of this contribution, or to allow others to do so, for U.S. Government purposes. Los Alamos National Laboratory requests that the publisher identify this article as work performed under the auspices of the U.S. Departmentof Energy. Los Alamos National Laboratory strongly supports academic freedom and a researcher's right to publish; as an institution, however, the Laboratory does not endorse the viewpoint of a publication or guarantee its technical correctness. 


\section{Neutron Propagation in Atmosphere}

John Leacock, PhD

15 October 2014

Los Alamos National Lab

for

Institute for Defense Analyses 
Create, deliver, and support innovative detection and energy-projection systems for remote applications in space and around the world.

\section{VELA Satellite, 1963}

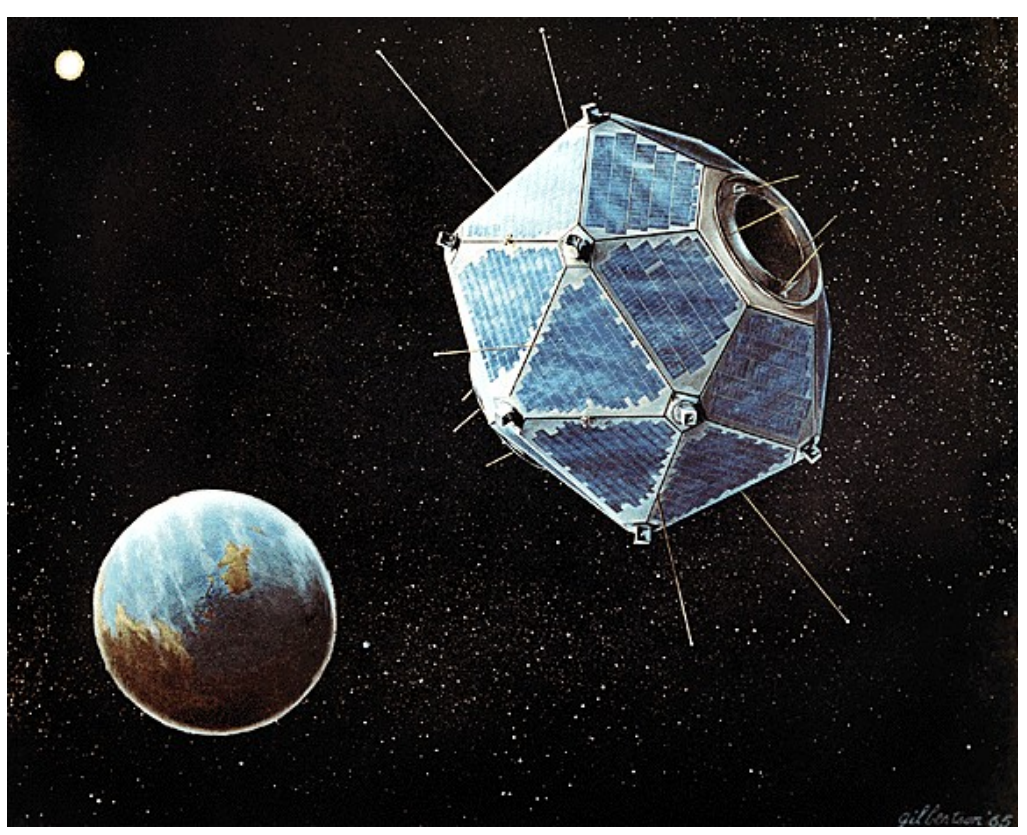

Badger 001 test, 1953

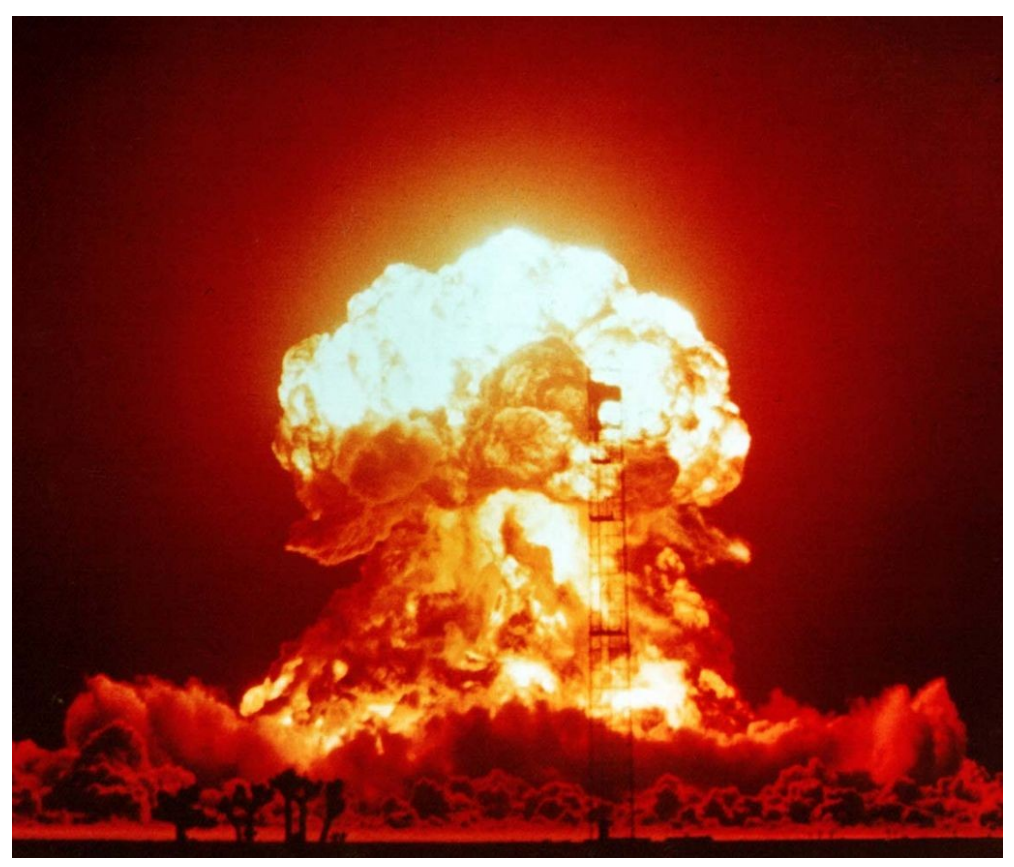




\section{USNDS - Project Description}

The United States Nuclear Detonation (NUDET) Detection System (USNDS) is designed to detect, locate, and report nuclear detonations (NUDETs) in the earth's atmosphere and near space in near real-time.

Nuclear Test Ban Treaty Monitoring - "Trust but verify"

USNDS uses space based systems on GPS (MEO) satellites and GEO satellites.

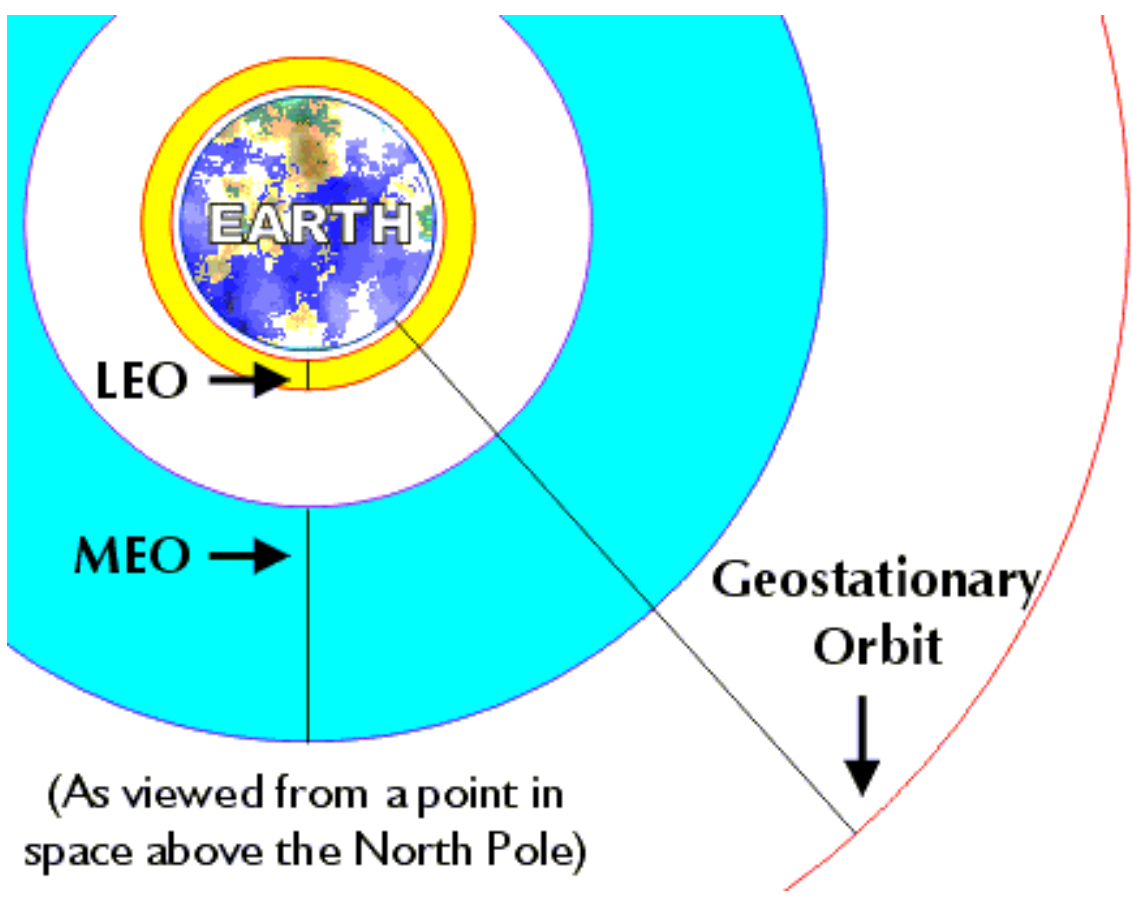




\section{USNDS - DIORAMA}

USNDS System Performance Modeling Tool with a focus on characterization of SNDD instrument performance. Sponsored by NA-22

Replaces existing fractured SNDD performance modeling tools with consolidated approach

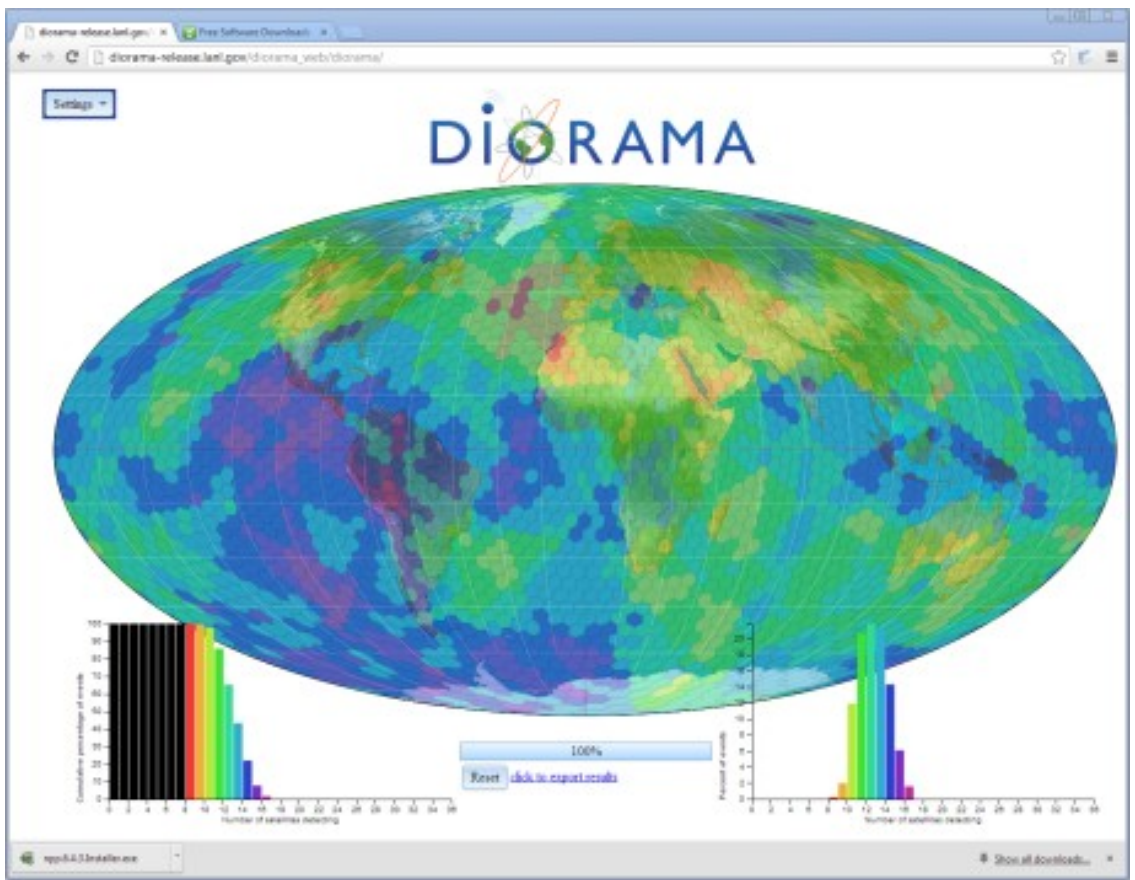

Web based tool - color coded map shows coverage capabilities. Performance for nonoperational instruments shown.

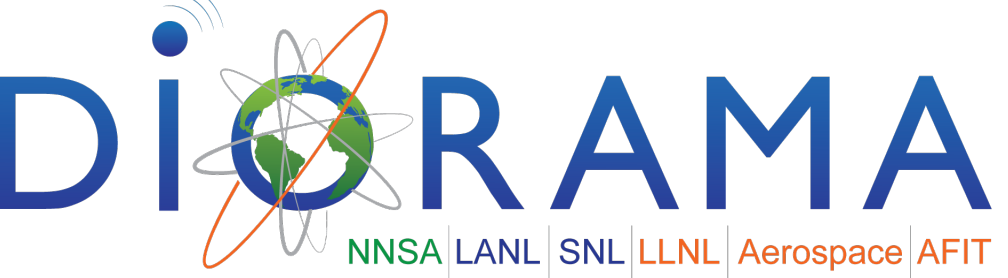

Distributed Infrastructure Offering Realtime Access to Modeling and Analysis 
Since a NuDet test of the satellite systems is impossible, the system must be evaluated some other way.

Diorama provides that functionality by simulating the response of the entire USNDS (detectors, communication, analysis) to a broad range of NuDets.

There are $\sim 24$ GPS satellites in orbit now, what if we changed that number?

What if we changed a detector on board?

How would the coverage change?

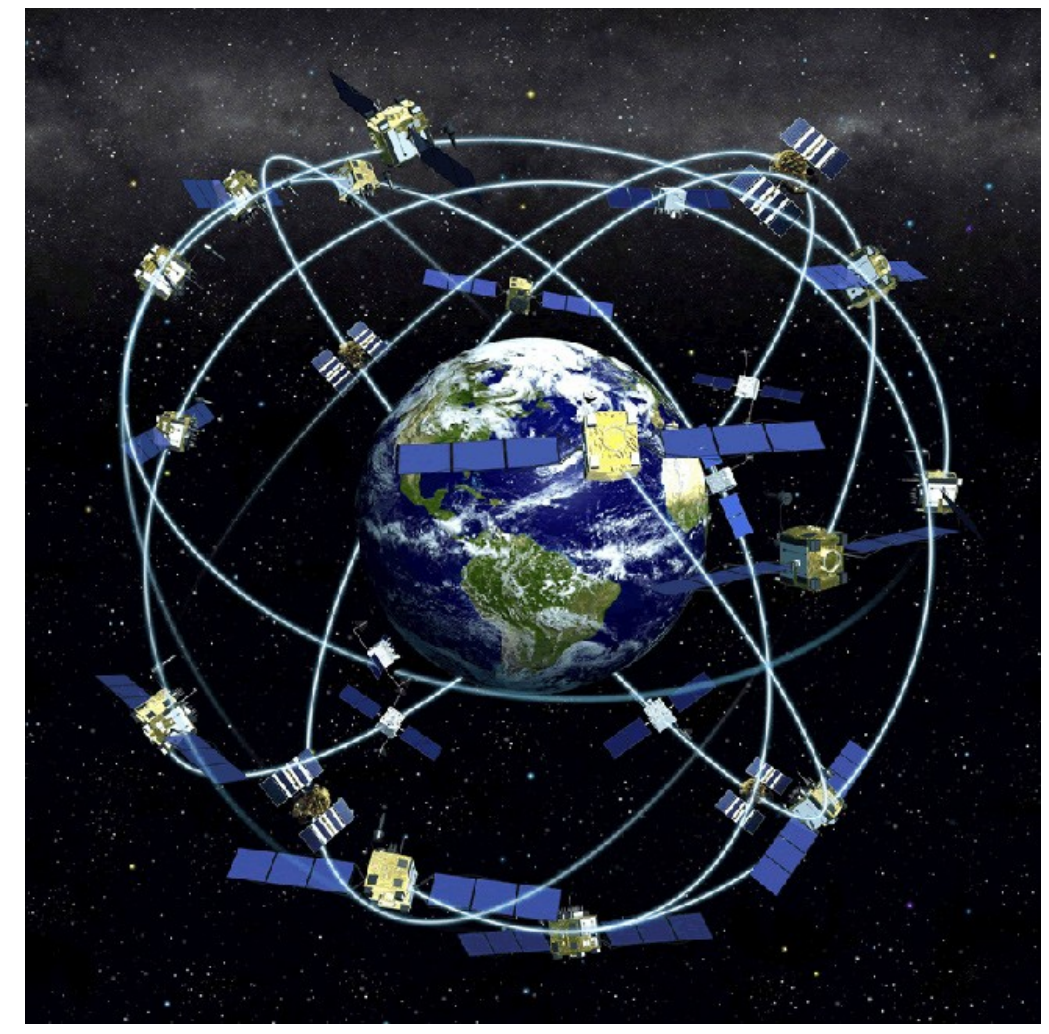




\section{NuDet - Phenomenologies}

Xrays - heat of explosion, similar to a light bulb at high energy

Gammas - fission and fusion energy release

Neutrons - fission/fusion byproduct and sustainer

Optical - flash of light

EMP - xrays/gammas ionize atmosphere, create wave of electrons

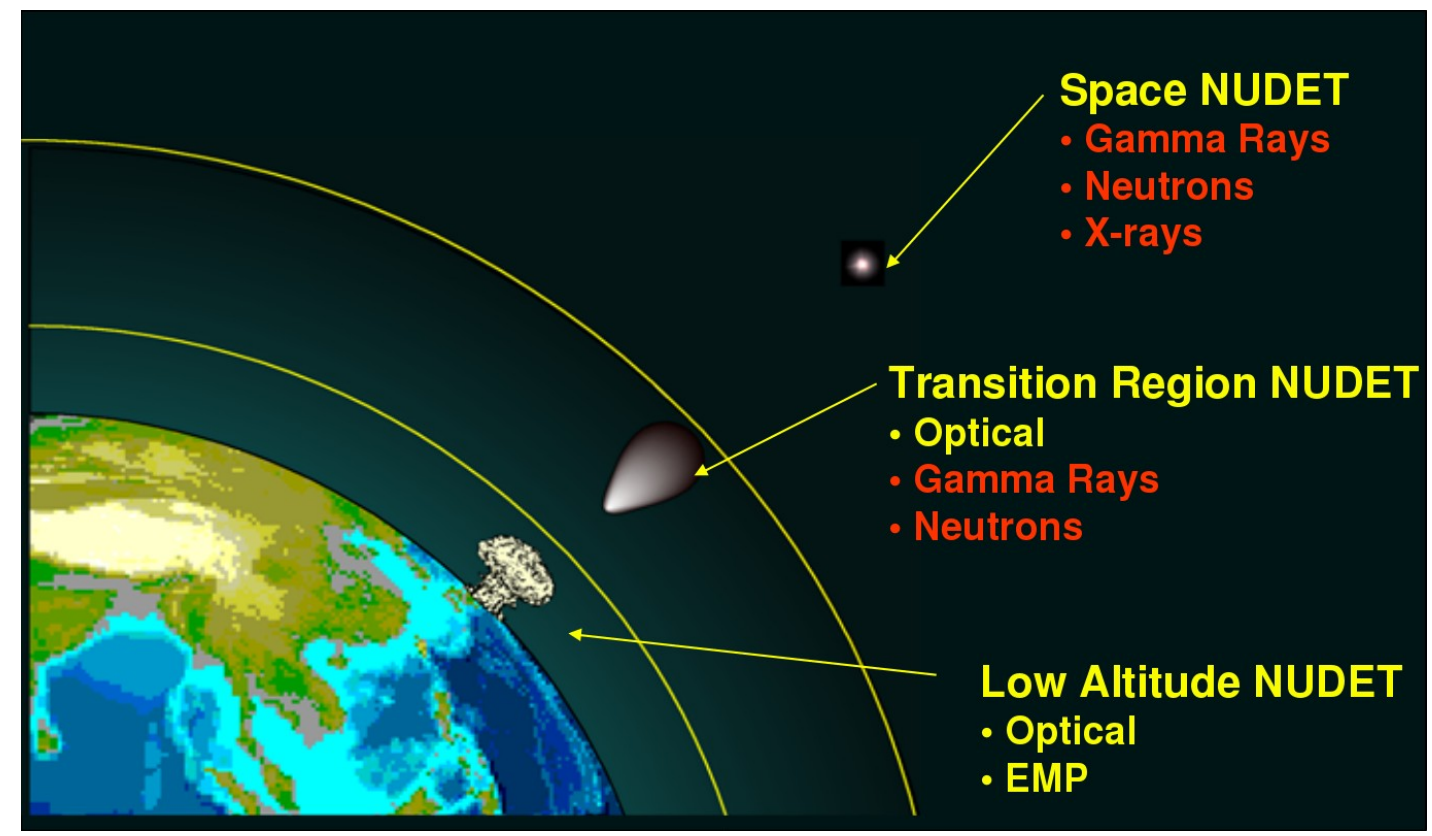

- Los Alamos

NATIONAL LABORATORY

Operated by Los Alamos National Security, LLC for the U.S. Department of Energy's NNSA

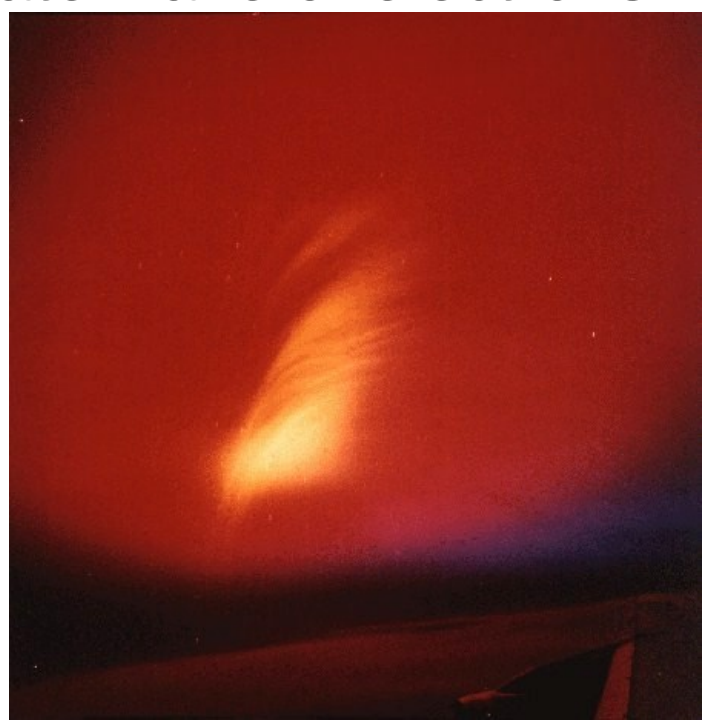

Artificial aurora caused by ionized particles in atmosphere as a result of a high altitude nuclear test. 


\section{Subatomic Particle Refresher}

An understanding of the structure of an atom and forces involved is necessary to proceed.

An atom is composed of three particles.

\begin{tabular}{lll} 
& Charge $[\mathrm{e}]$ & Mass $\left[\mathrm{MeV} / \mathrm{c}^{\wedge} 2\right]$ \\
\hline proton & +1 & 938.2 \\
\hline neutron & 0 & 939.5 \\
\hline electron & -1 & 0.511
\end{tabular}

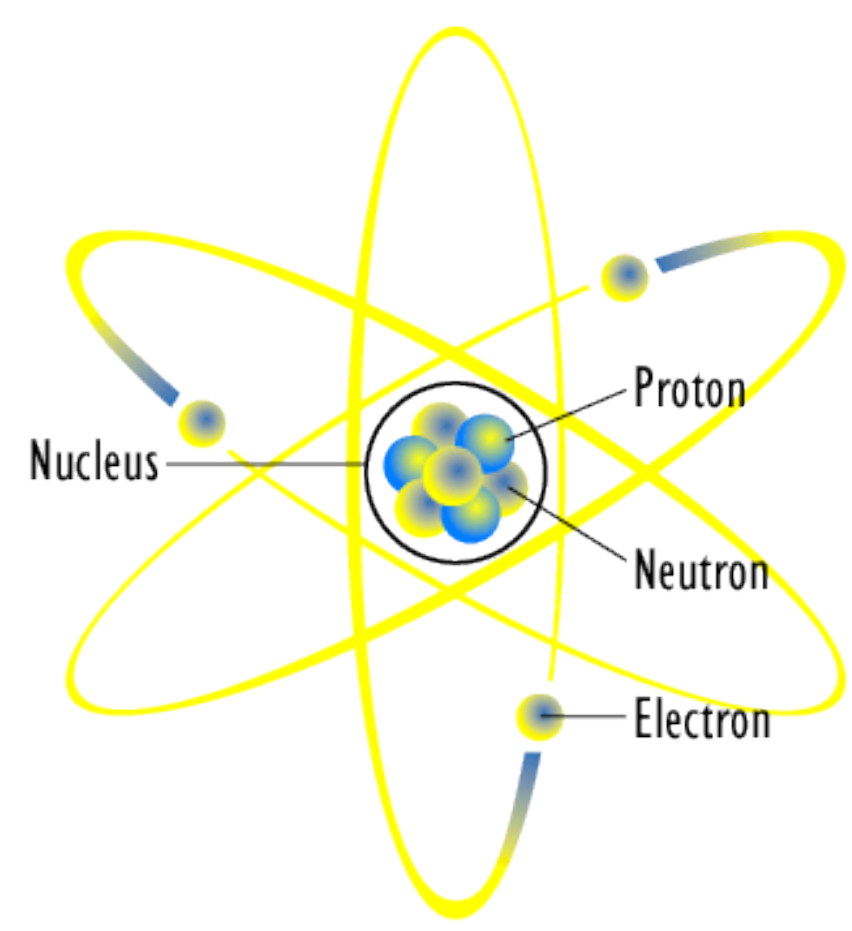

What holds a nucleus with net positive charge together? 
All particle interactions are defined by one of the following four fundamental forces. For the purposes of this simulation gravity and the weak force are ignored.

Gravity - dominates over galactic scales

Electromagnetic - binds the electrons to the nucleus

Weak - mode in which the neutron decays

Strong - binds the protons and neutrons within a nucleus, fission/fusion 
Fundamental Forces Refresher

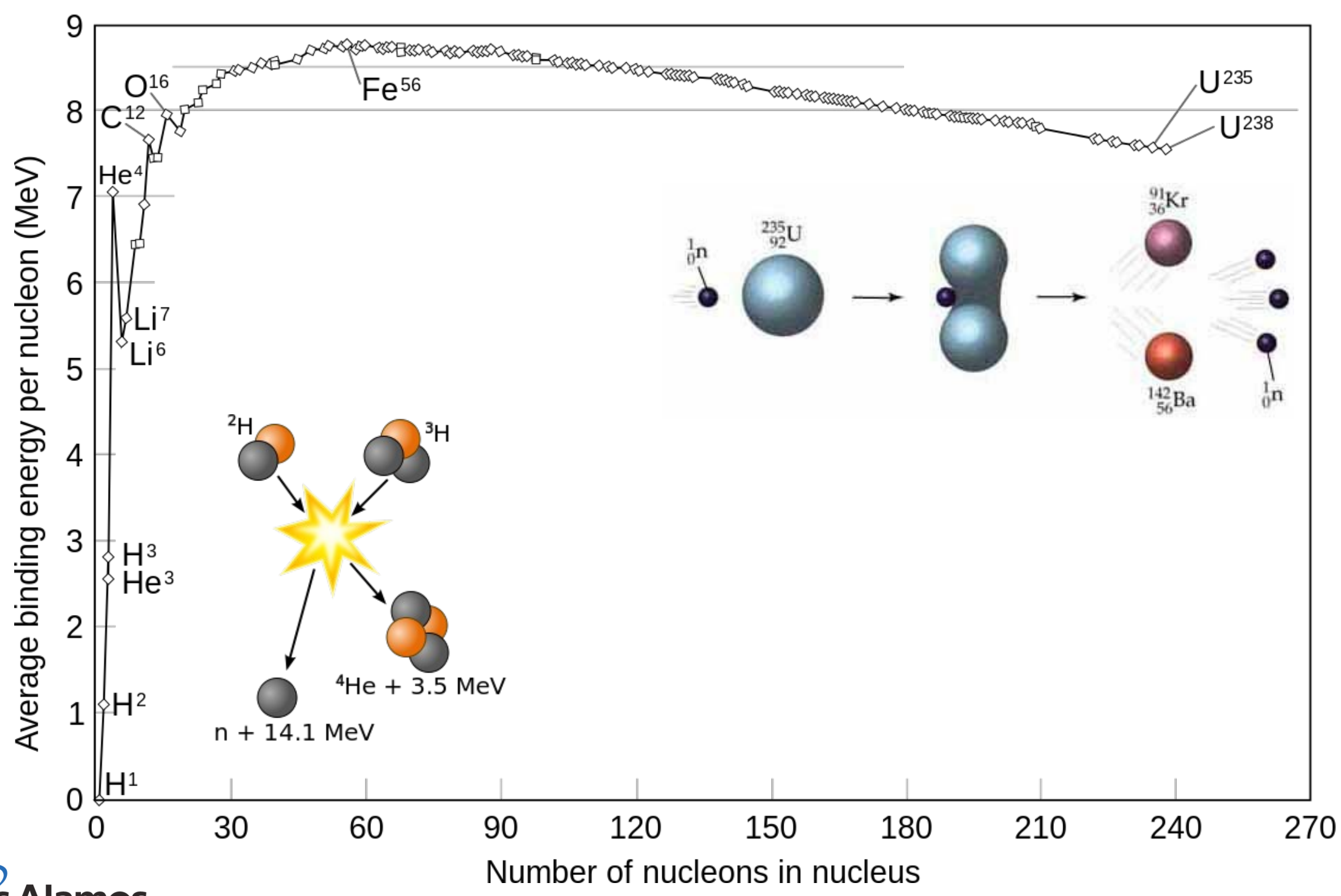




\section{Neutron Current Research}

The free (unbound) neutron is not a stable particle and measuring its lifetime is a hot topic in physics.

\section{WEIGHTED AVERAGE}

$880.1 \pm 1.1$ (Error scaled by 1.8)

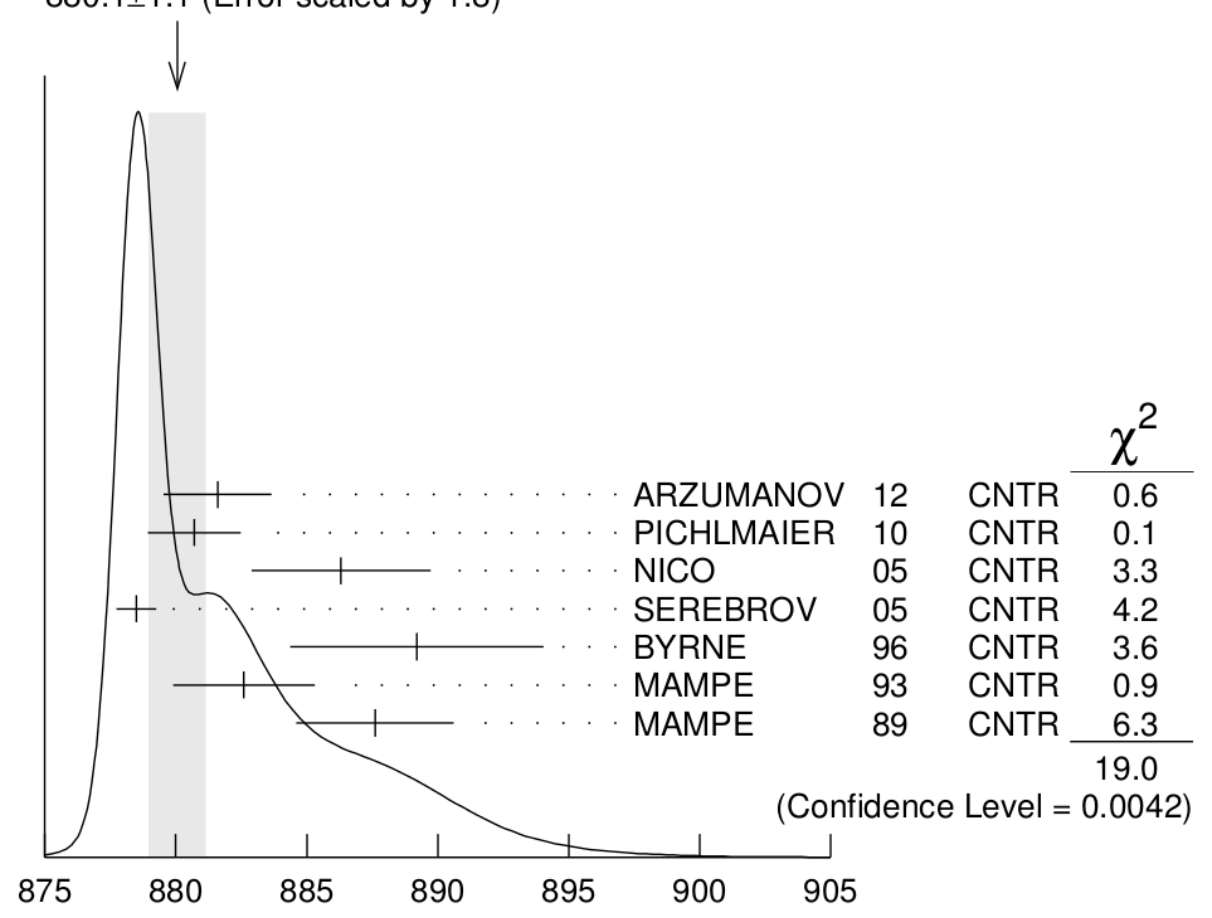

Because of the timescales involved, DIORAMA is not concerned with neutron decay but it is interesting that such a fundamental parameter is relatively unknown.

neutron mean life (s) 


\section{Sources of Neutrons}

Spallation Neutron Source at ORNL, used for scientific experiments

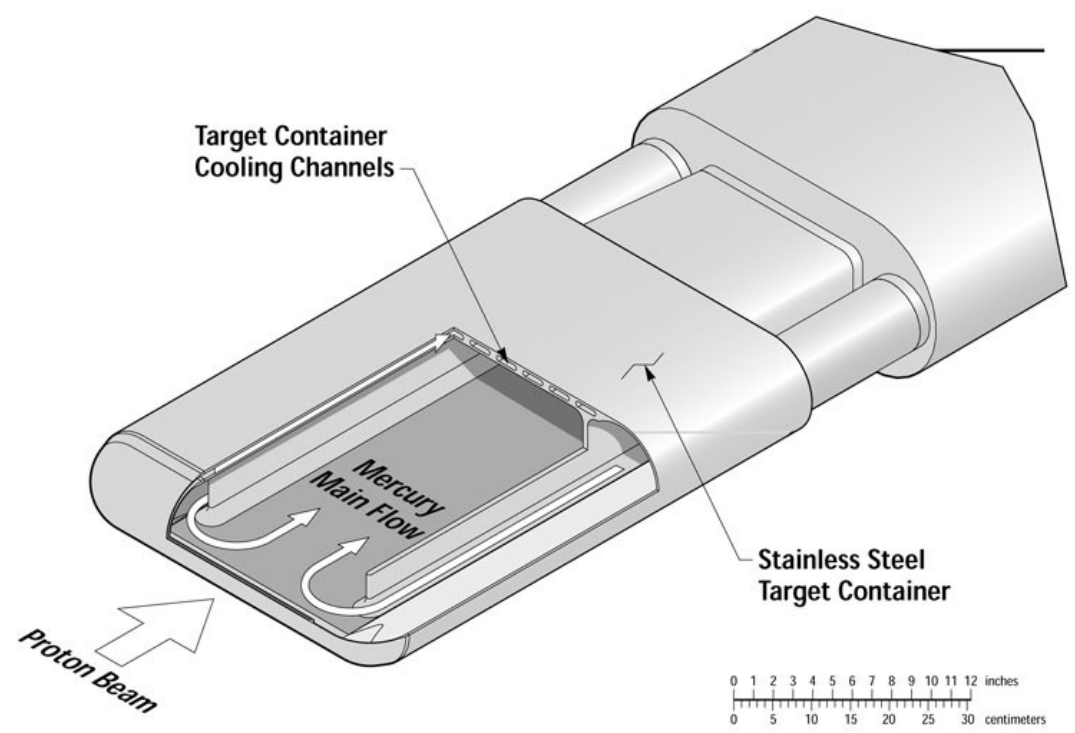

Fission in nuclear reactors

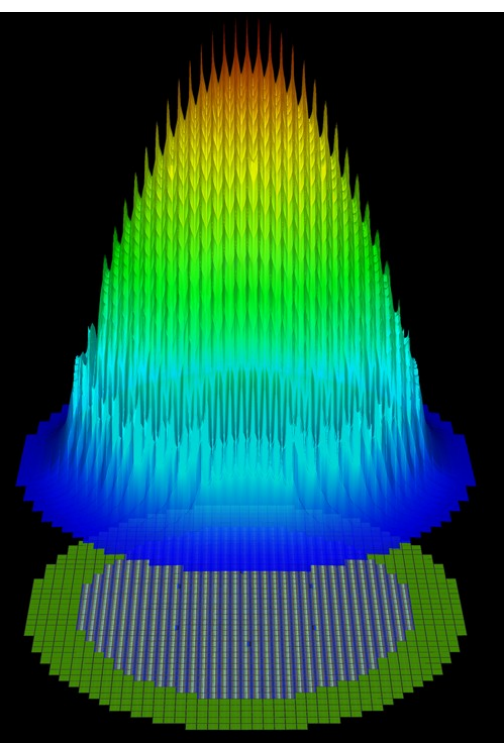

Nuclear detonations, "no innocent neutrons in space"

- Neutrons in space are a smoking gun for nefarious activity 


\section{NuDet Phenomenologies - Neutrons}

Nuclear detonations release energy in the form of particles. For this talk we are concerned with the neutrons.

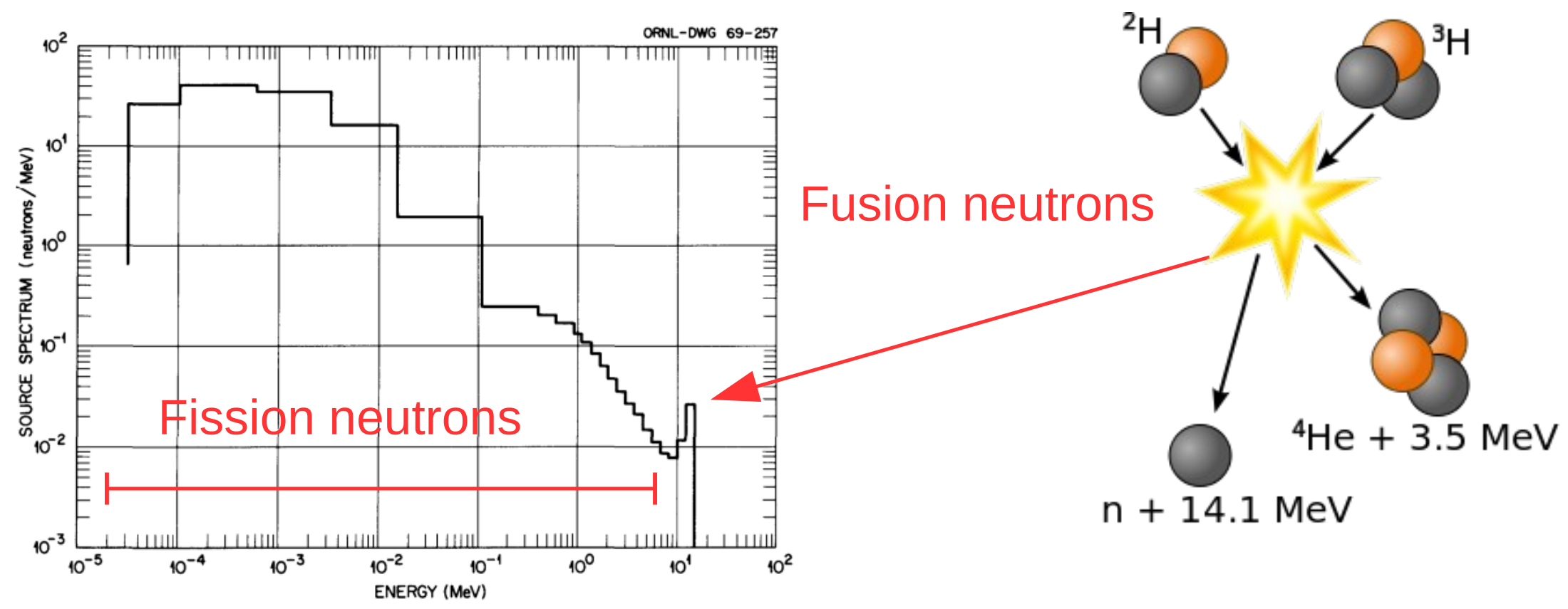

The source energy for a Typical Thermonuclear Weapon 


\section{Neutrons - Cross Sections}

A cross section is an effective area of interaction probability measured in barns. 1 barn is $1 \mathrm{e}-24 \mathrm{~cm}^{\wedge} 2$
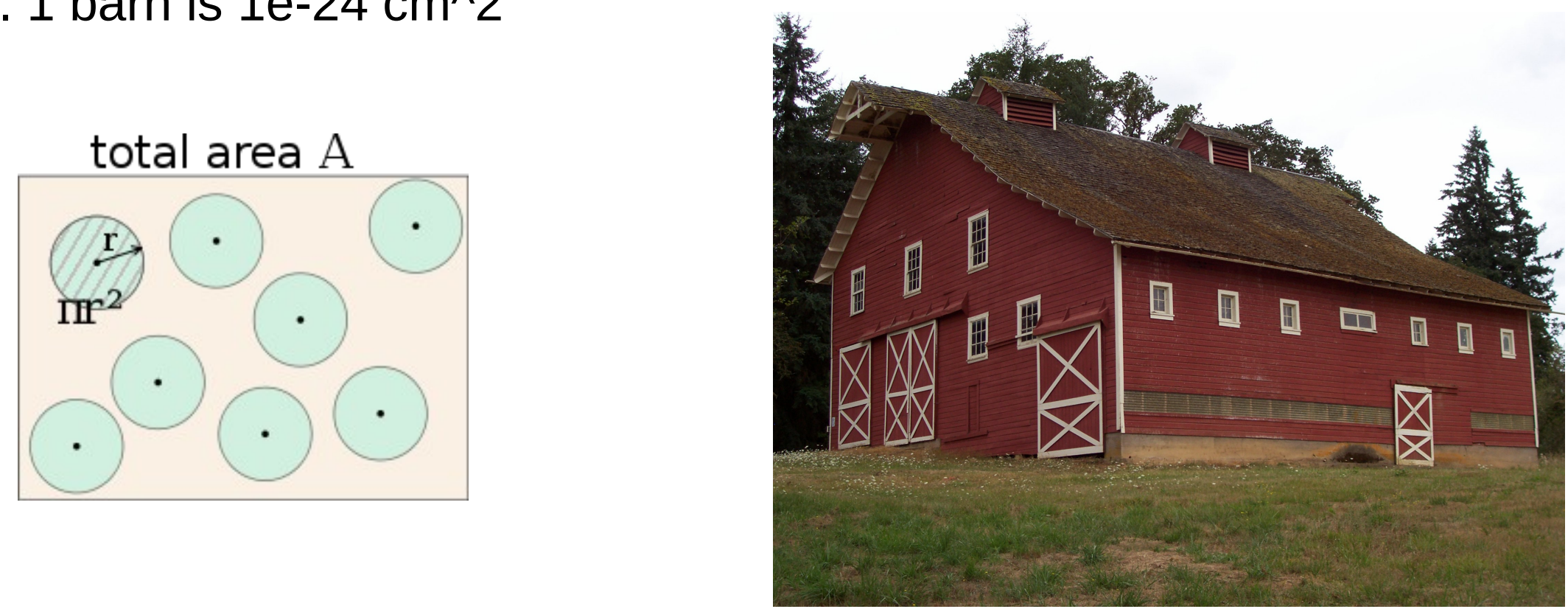

A simple view of cross section is how big a particle appears to the interacting particle. This value has little to do with the actual size of interacting particles. 
The cross section of interaction of a neutron typically decreases with increasing neutron energy.

A moderator is typically used to slow the neutron so it will have a higher probability of interacting.

Note Helium-3 has a very high absorption cross section.

For DIORAMA we are interested in $200 \mathrm{eV}$ to $14.5 \mathrm{MeV}$ neutrons.

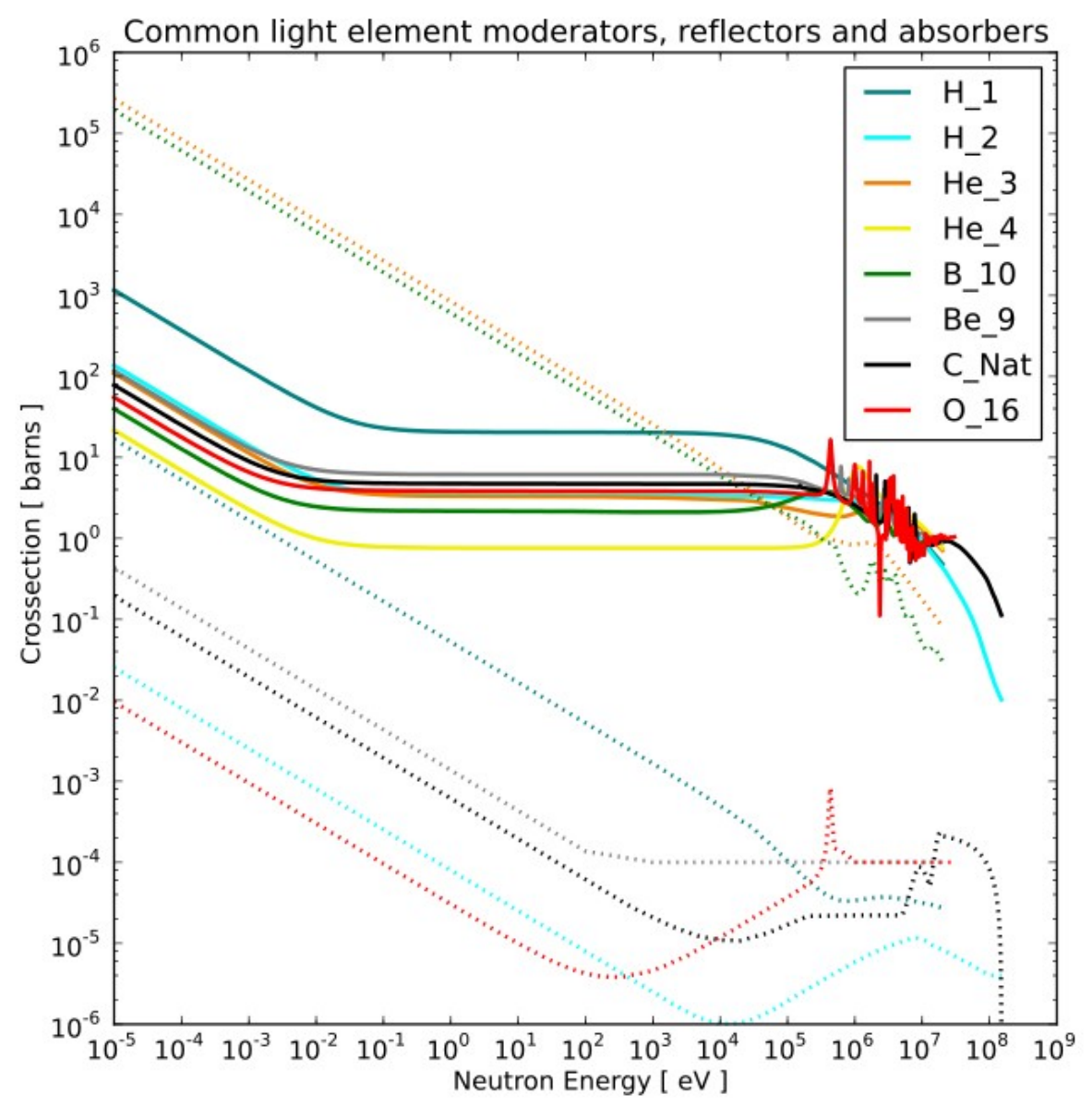

Cross sections of light elements, Solid lines - scattering Xs Dotted lines - absorption xs 


\section{Neutrons - Detection}

Neutrons are relatively difficult to detect. With charge zero, a neutron could pass through several centimeters of lead without interacting.

Exploit Helium-3 or Lithium-6 or Boron-10 absorption cross sections.

A neutron absorbed by an $\mathrm{He}-3$ atom creates $\mathrm{H} 3+\mathrm{p}+0.764 \mathrm{MeV}$.

These particles ionize the gas and that charge is read out.

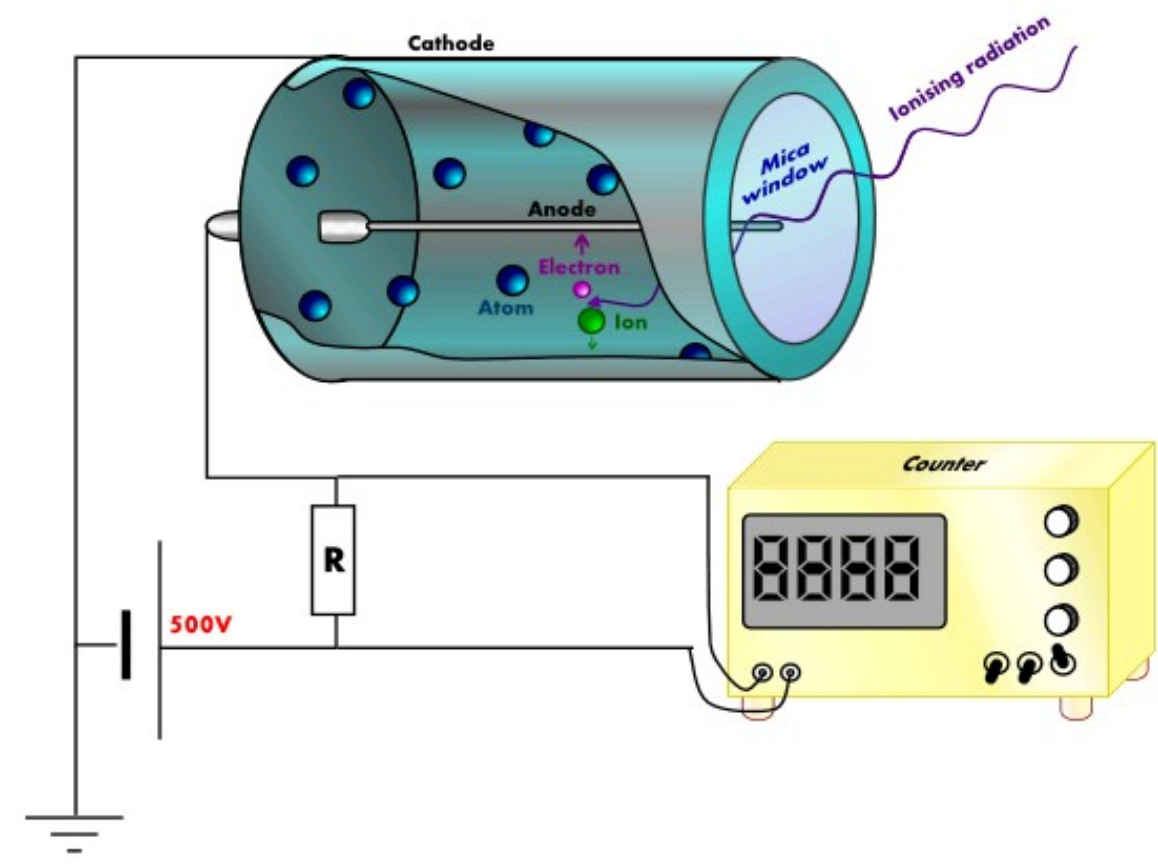


DIORAMA simulates the phenomenology's source, propagation, and detection in a probabilistic manner. Each probability is randomly sampled from a probability distribution function.

Xray is emitted from simulated NuDet

Source: With what energy and direction?

Propagation: Does it make it to detector?

Detection: Given the energy what is the probability of detection?

Each of these probability distributions is sampled simultaneously and these are reflected in the probability distribution of the final performance metrics. 


\section{Monte Carlo}

Let's say you want to know the probability of winning a game of solitaire.

Combinatorics could provide an answer if computers were capable.

A simpler method exists though. Play a "large" number of games of solitaire and tally which games were won and which were lost.

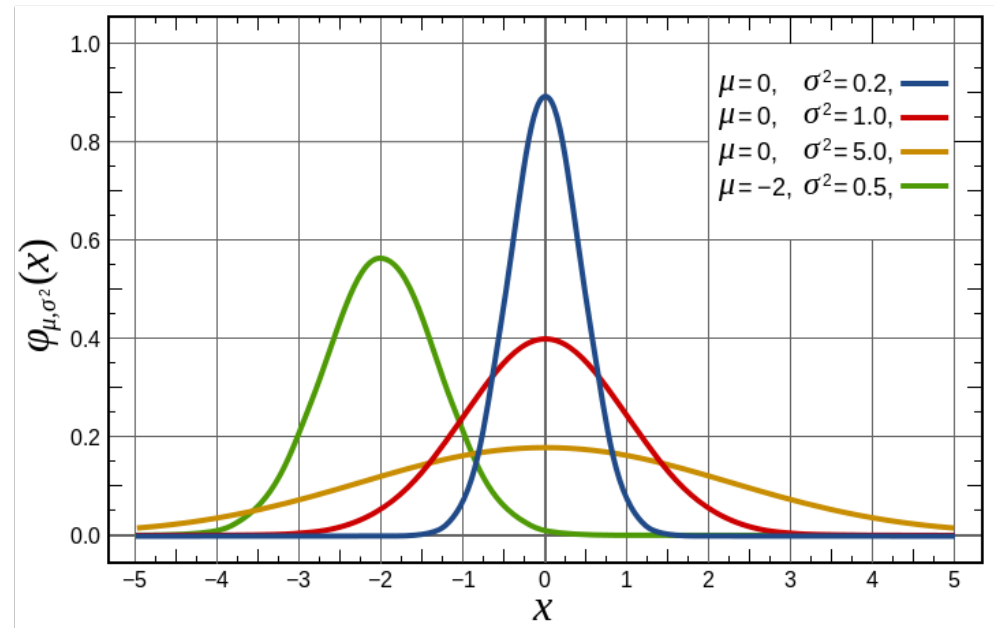

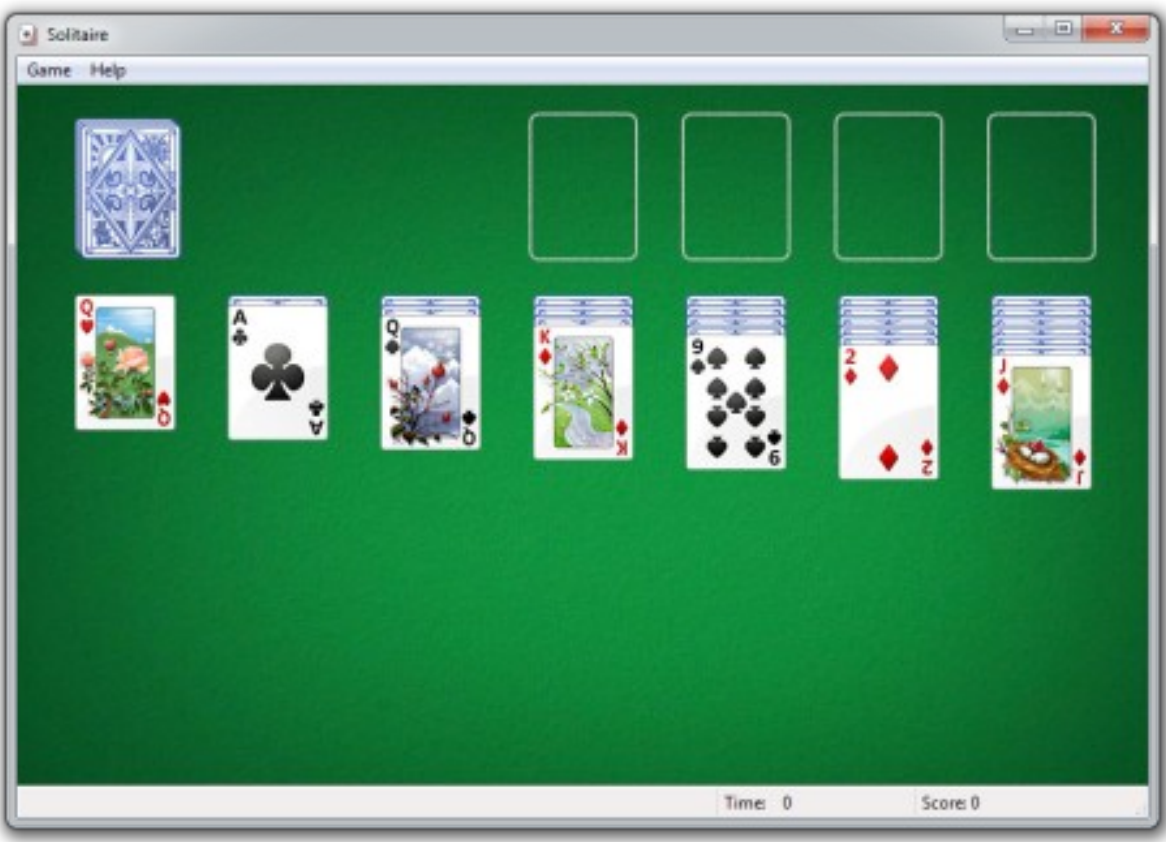

The result will be a "bell curve" and from the width of the distribution and number of trials one can calculate the uncertainty of the result. 
Must know role of atmosphere in neutron propagation to determine yield of weapon (analysis) or the capabilities of the system (simulation).

For example, a detonation on the surface of the earth will have different particle signatures than a detonation at $30 \mathrm{~km}$.

Each detonation is unique and the effect of the atmosphere contributes to the variety of possible signatures. 


\section{DIORAMA - Neutron Propagation}

Given a source energy neutron we need a time distribution at a GEO location.

Transport matrix gives the probability a neutron with given source energy will arrive at GEO at a certain time.

Each row is a Time of Arrival probability distribution function for a single energy bin.

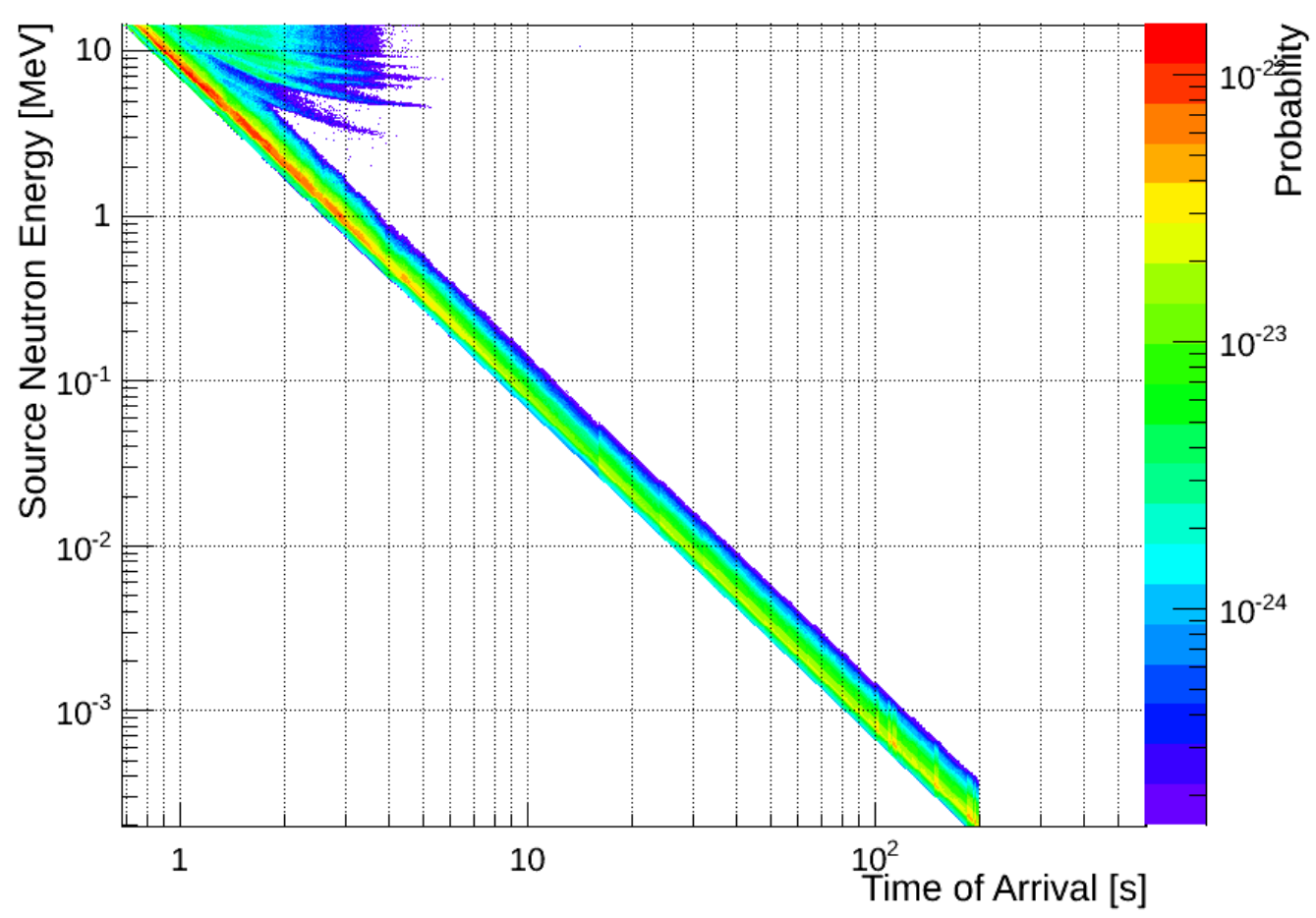




\section{DIORAMA - Neutron Propagation}

Current state of the art neutron propagation requires gigabytes of matrices to turn a source matrix into a Time of Arrival distribution.

Source

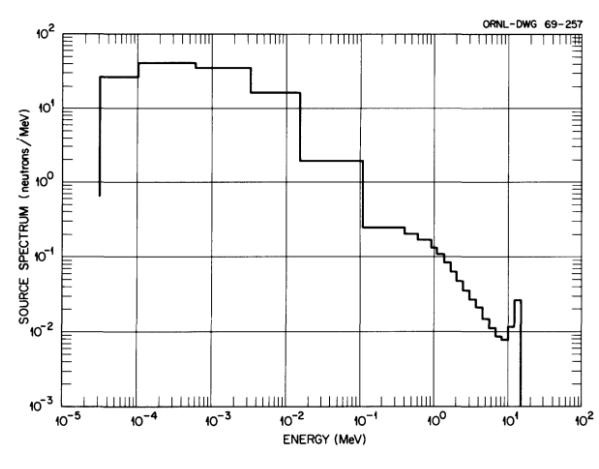

Transport Matrix

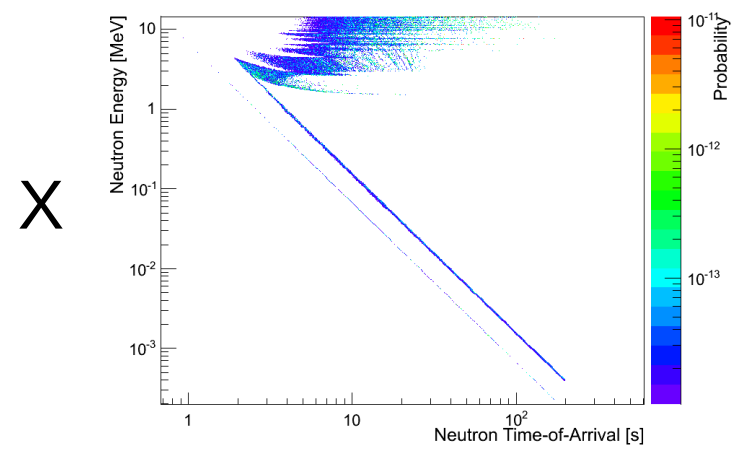

TOA distribution

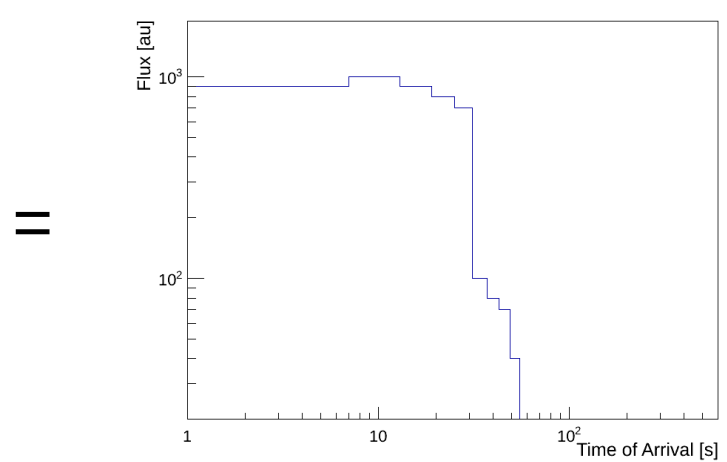

We need a PDF for each energy, altitude, angle, and multiplicity. In total that is $\sim 20 \mathrm{~GB}$ of matrices.

An empirical function would greatly reduce the storage and processing time needed to transport neutrons in atmosphere. 


\section{Photon Propagation}

Photons are also released from nuclear detonations. However, their behavior is much better defined by deterministic methods.

The photons are wellbehaved and interact with elements in a manner described by the functions similar to the one shown here.

If a photon interacts with an element en route to the detector it is assumed lost.

That is not the case for neutrons.

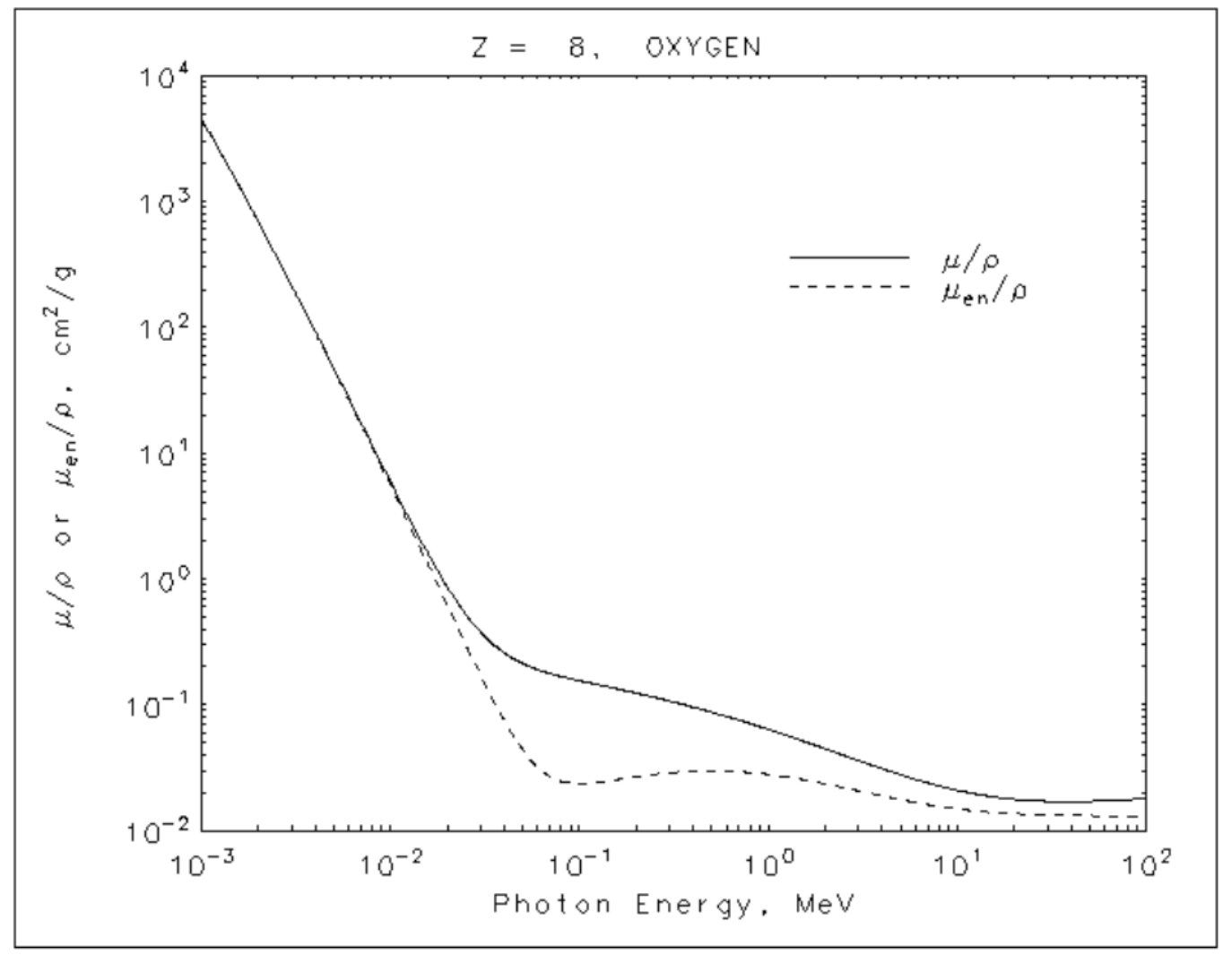


"Geant4 is a toolkit for the simulation of the passage of particles through matter. Its areas of application include high energy, nuclear and accelerator physics, as well as studies in medical and space science."

Fermilab experiment Geant4 sim.
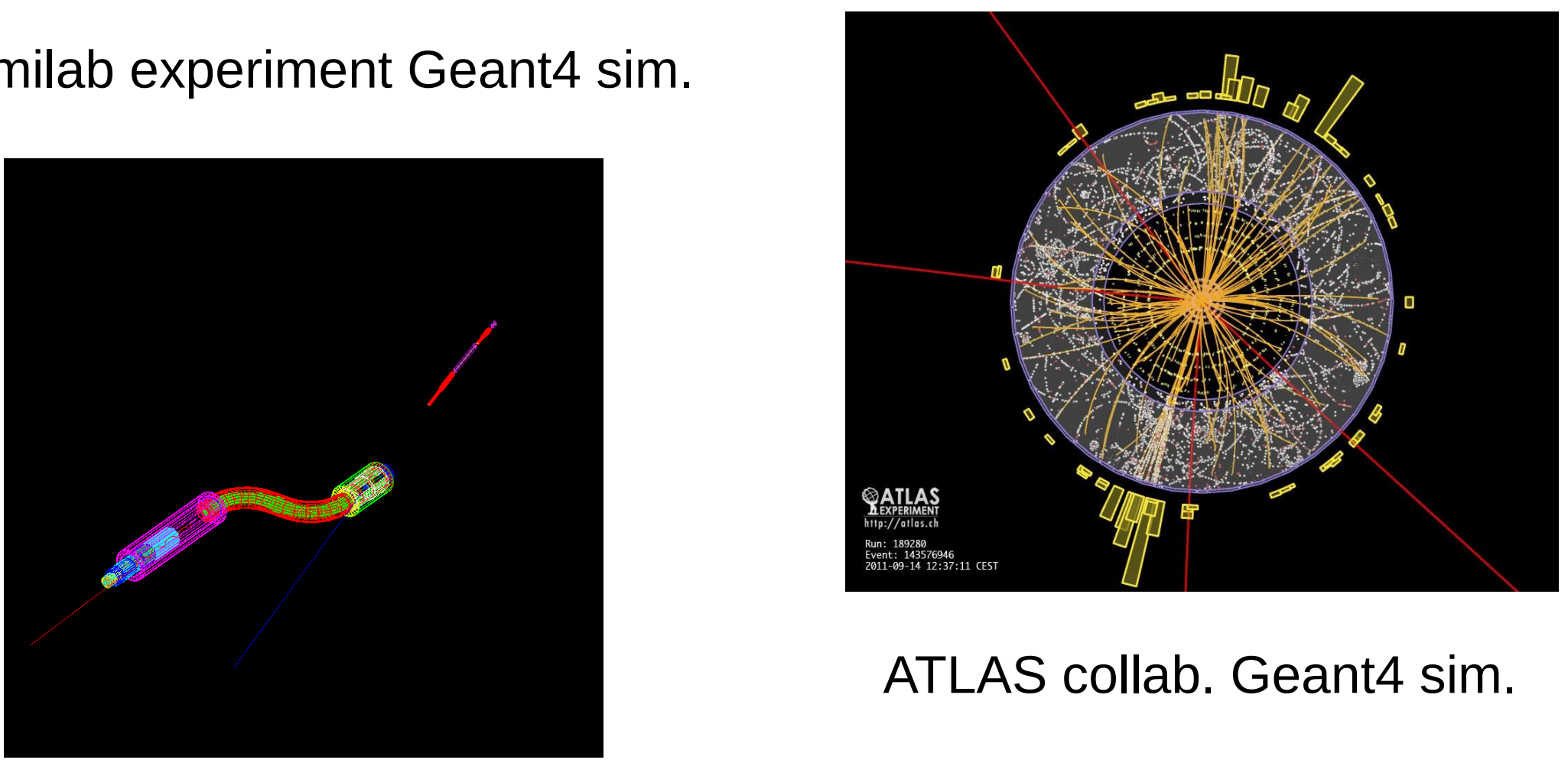

ATLAS collab. Geant4 sim. 


\section{Geant4 - Geometry}

The earth is modeled as a sphere of $\mathrm{SiO} 2$ with radius $6738 \mathrm{~km}$.

The atmosphere is taken from the NRLMSISE-00.

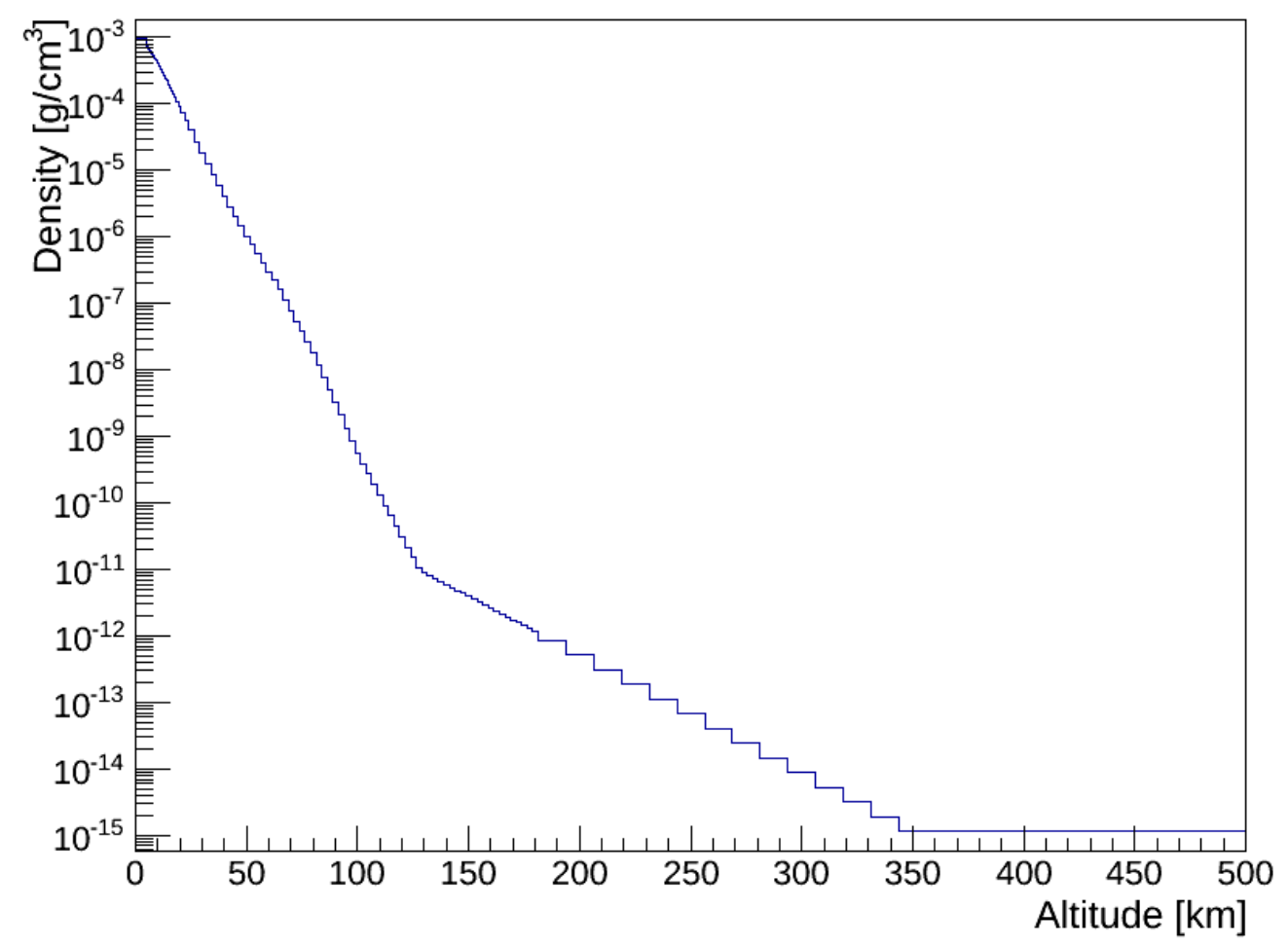

131 layers, thinner at lower altitudes 


\section{Geant4 - Atmosphere Makeup}

In the Geant4 model the atmosphere contains several isotopes that contribute to the overall cross section of the air.

\begin{tabular}{|l|l|} 
& Elemental abundance [\%] \\
\hline Nitrogen & 78.45 \\
\hline Oxygen & 21.08 \\
\hline Argon & 0.47 \\
\hline
\end{tabular}

These are the common constituents of the atmosphere.
These are the isotopic constituents.

\begin{tabular}{|l|l|l|} 
& Elem abund [\%] & Molar mass $[\mathrm{g} / \mathrm{mole}]$ \\
\hline N14 & 78.16 & 14.00 \\
\hline N15 & 0.29 & 15.00 \\
\hline O16 & 21.03 & 15.99 \\
\hline O17 & 0.051 & 16.00 \\
\hline Ar36 & $1.6 \mathrm{e}-3$ & 35.97 \\
\hline Ar38 & $2.8 \mathrm{e}-4$ & 37.96 \\
\hline Ar40 & 0.468 & 39.96 \\
\hline
\end{tabular}


The cross section of air is dominated by nitrogen and oxygen but the other isotopes subtly shift the curve and peaks.

The effect has not been quantified but in order to validate cross sections all

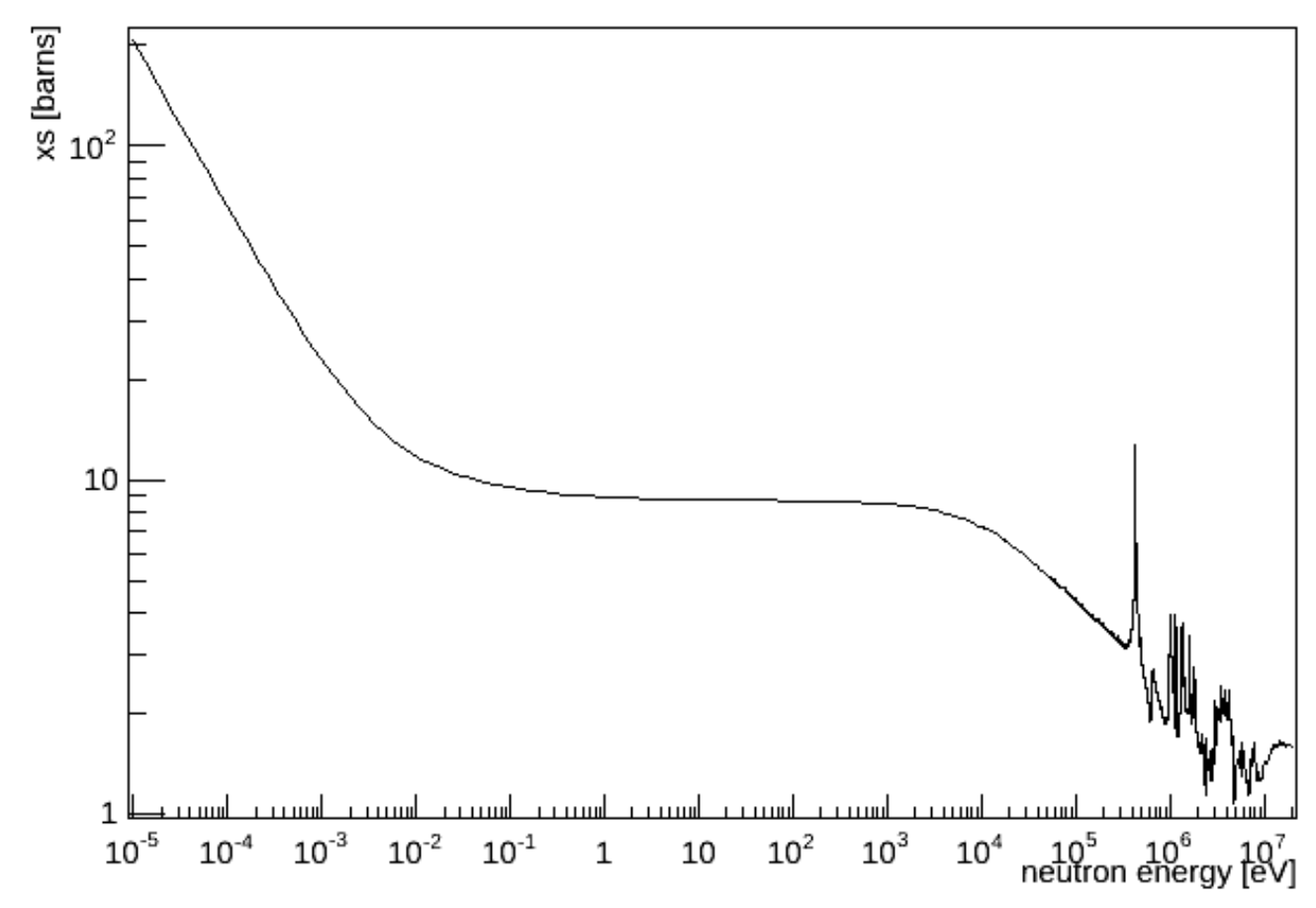
isotopes must be included. 


\section{Geant4 - Simulation Details}

5e11 neutrons were required to acquire the statistics necessary for an acceptable uncertainty.

In Geant4, individual neutrons are tracked through their propagation in atmosphere and at each interaction point. This results in a huge number of calculations that require parallelization.

In total 6 terabytes of data were generated. For a total of 13 million hours of computation time.

In order to analyze the Geant4 outputs, a parallel analysis code was developed. This program is memory intensive $\sim 60 \mathrm{~GB}$ and completes in 20 hours. 
A source of neutrons was simulated for a range of altitudes $(10 \mathrm{~km}$ to $20,000 \mathrm{~km})$.

A source of neutrons at $10 \mathrm{~km}$ behaves very different than a source at $20,000 \mathrm{~km}$. The thickness of the atmosphere attenuates and disperses the neutrons at low altitudes but those effects are relatively absent from high altitudes.

At altitudes above the atmosphere $(\sim 100 \mathrm{~km})$ reflection becomes a significant contribution. Neutrons initially downward directed bounce off of the atmosphere and back toward the detectors 
Geant4 also includes thermal excitation of neutrons as it affects the cross sections, a "room temperature" neutron has $0.025 \mathrm{eV}$ of kinetic energy and can easily gain or lose a significant fraction of its energy from the environment.

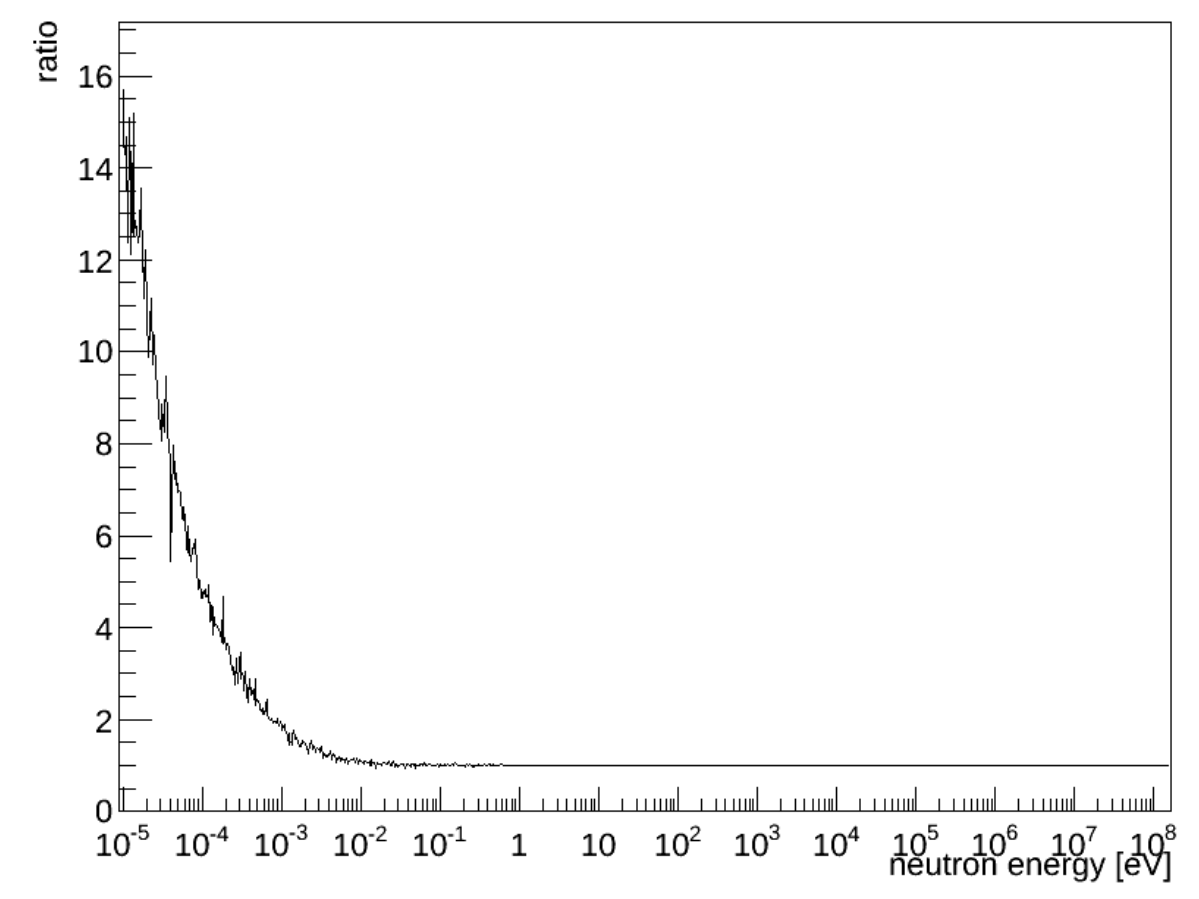

This ratio is of the cross section for a $300 \mathrm{~K} / 0 \mathrm{~K}$ environment.

Notice the jitter on the ratio. That is due to thermal motion and is not present about $\sim 0.025 \mathrm{eV}$. 


\section{Geant4 - Geometry Validation}

Geometry contributes to the number of neutrons detected and its contribution was quantified and validated.

For each altitude and angle of detection (central angle $\beta$ ) the number of neutrons to make it to a detector ignoring the atmosphere and with a perfectly absorbing earth needed to be known.

$$
T=\frac{1}{4 \pi} \frac{r_{s}}{2 r^{3}}\left(r^{2}+r_{s}^{2}-r_{e}^{2}\right)
$$

$r=-r_{e} \cos \xi+\sqrt{r_{s}^{2}-r_{e}^{2} \sin ^{2} \xi}$

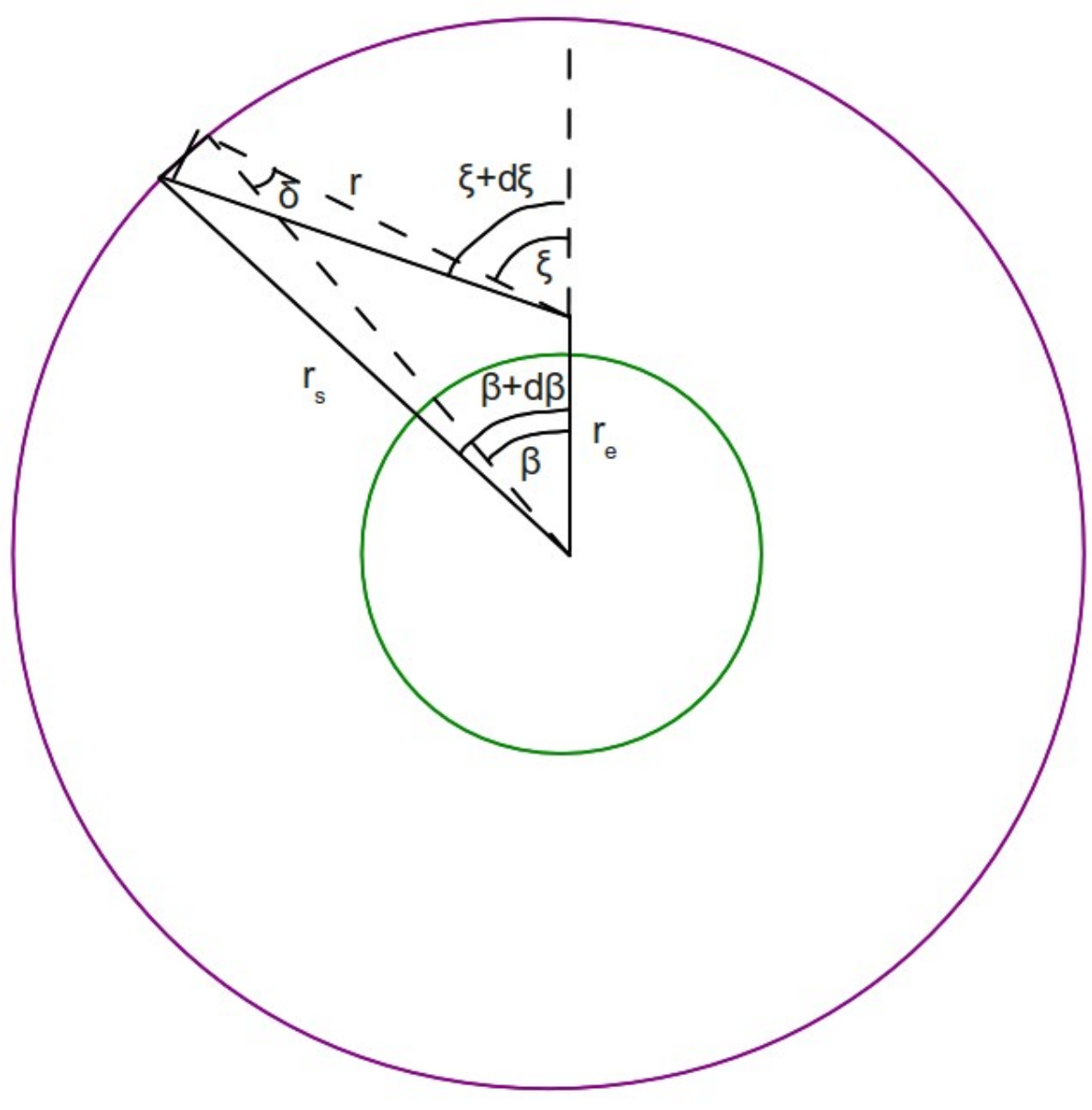




\section{Geant4 - Geometry Validation}

The equation depends on the source altitude, the detection altitude and the angle.

This equation agrees well with all input ranges and accounts for the occlusion due to the earth.

Again this is for no atmosphere and a perfectly absorbing earth.

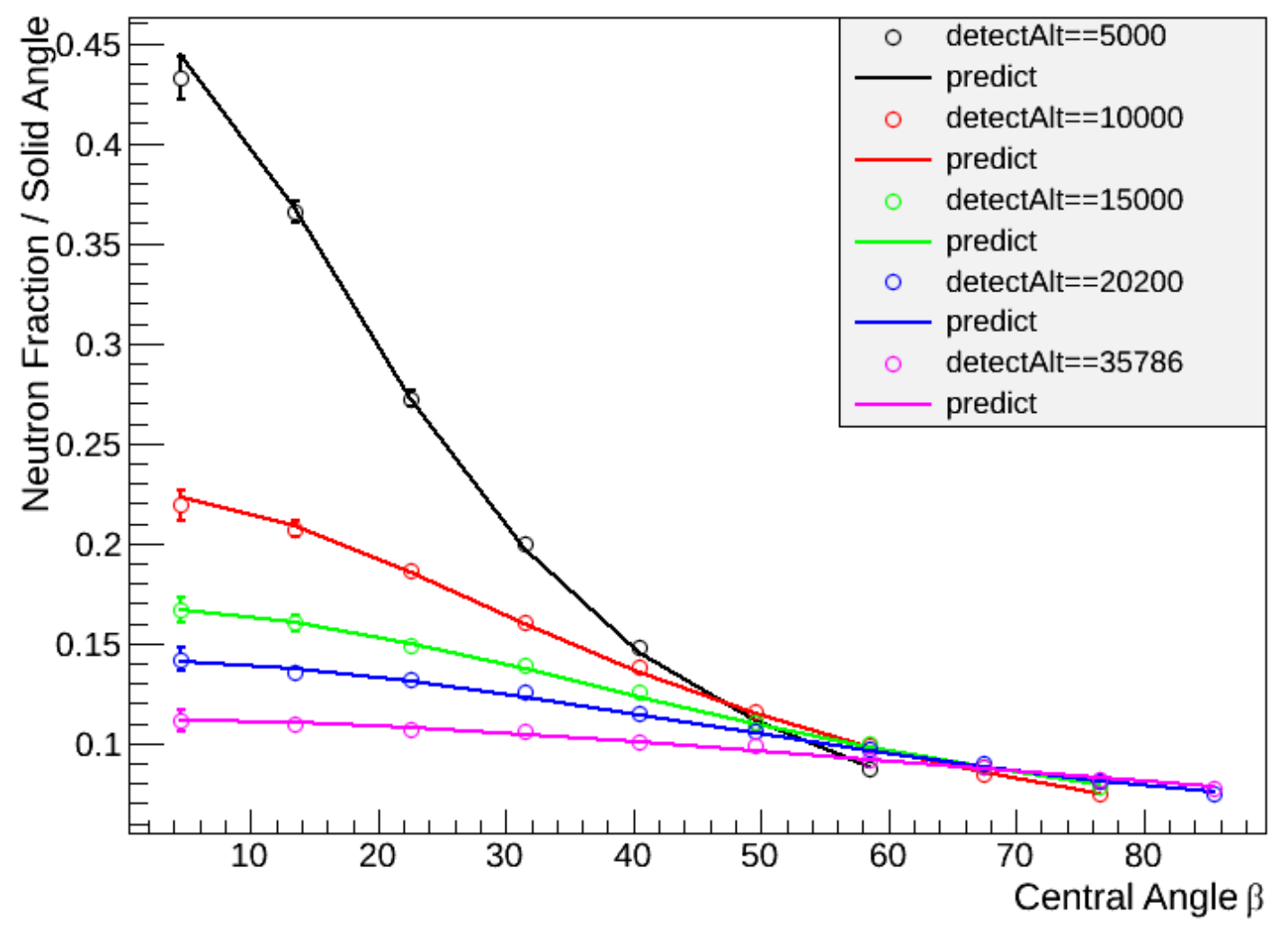


In order to validate that Geant4 was properly treating the cross sections, I wrote a script that read in the ENDF cross sections and applied it to a source of neutrons traveling through $12.05 \mathrm{~g} / \mathrm{cm} 2$ column density or from $30 \mathrm{~km}$ to $35786 \mathrm{~km}$.

This plot shows the ratio of the script to actual Geant4 output.

This is only for elastic scattering.

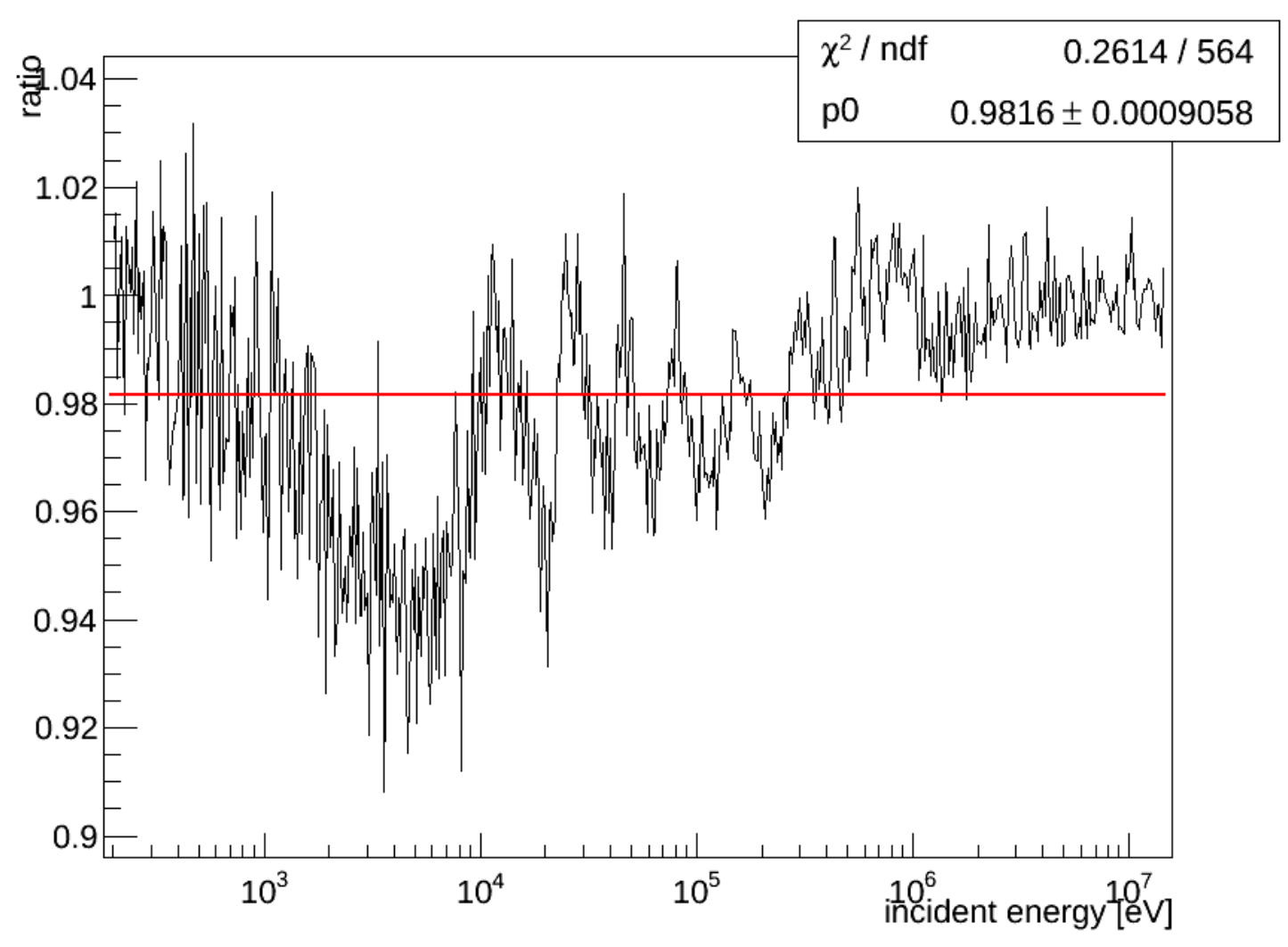




\section{Geant4 - Scattered N Results}

Since the goal is to find a functional form of the PDF, fits must be made to the data. One metric to plot against is the column density, which is just the amount of air between the source of neutrons and detector.

Here is a fit of scattered neutrons detected as a function of column density for a $50 \mathrm{~km} 1 \mathrm{keV}$ source.

The fit obeys the power law, which is expected for a sum of sequential exponentials.

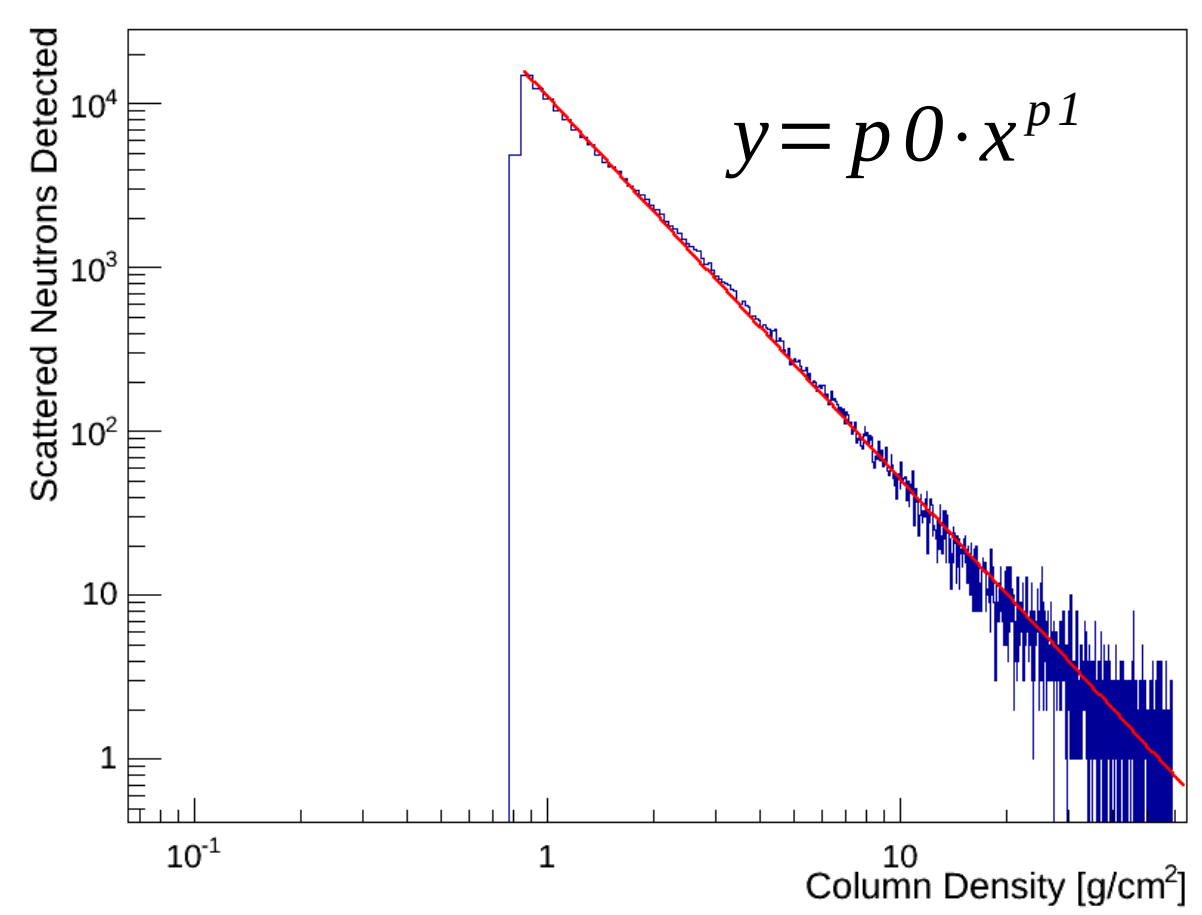


Expanding the plot by including more source energies and altitudes gives a glimpse of how the neutron transport functional form may look.

$$
y=p 0 \cdot x^{p 1}
$$

This fit is valid for all interesting energies but only $20-100 \mathrm{~km}$ source altitude.

Above $100 \mathrm{~km}$ the column density is too small to be of use for this plot. A better fit must be developed.

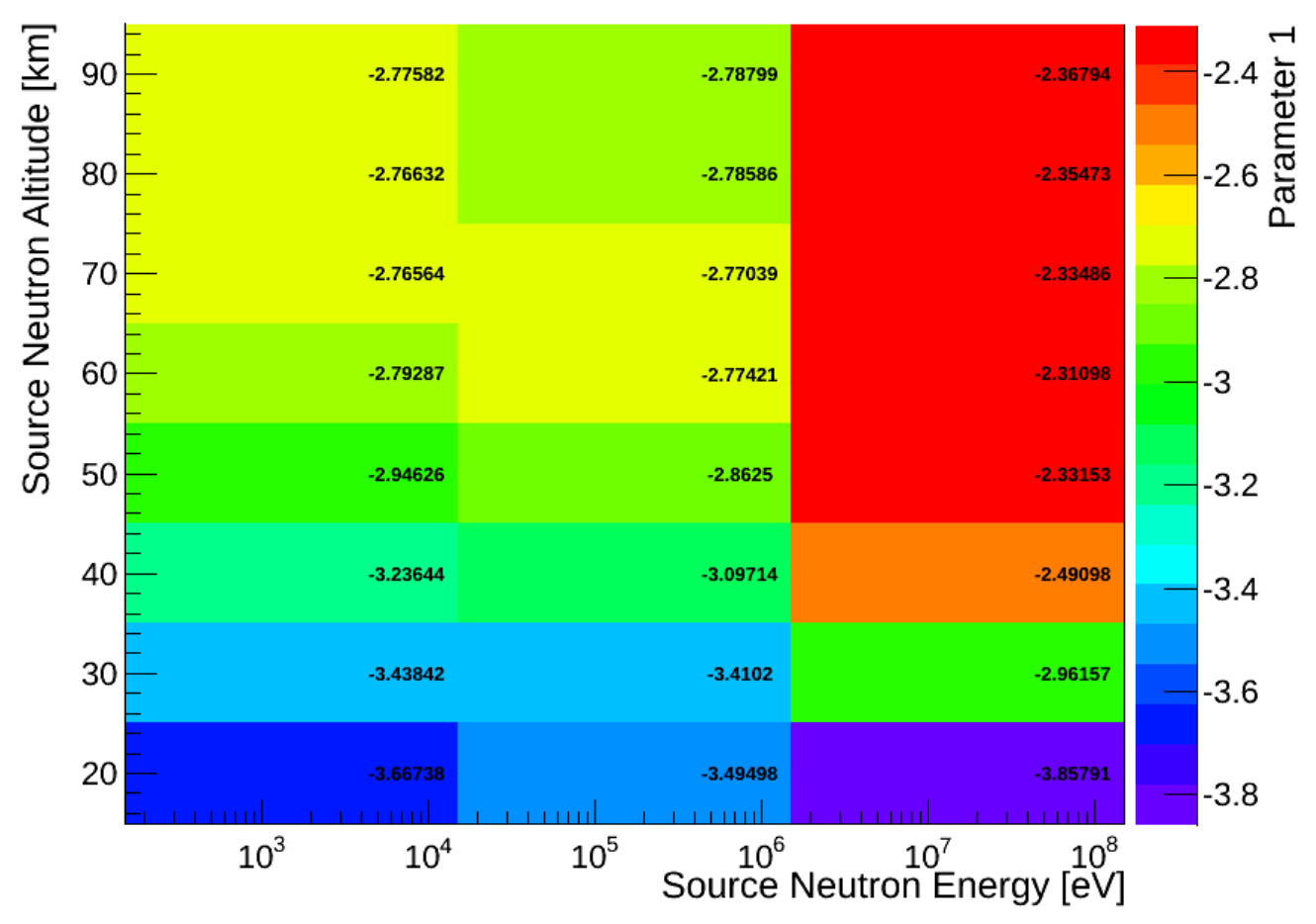


Need to replace lookup tables with empirically defined function

- Faster

- Portable

- Fewer computing resources

MCNP based neutron propagation in the atmosphere requires gigabytes of lookup tables

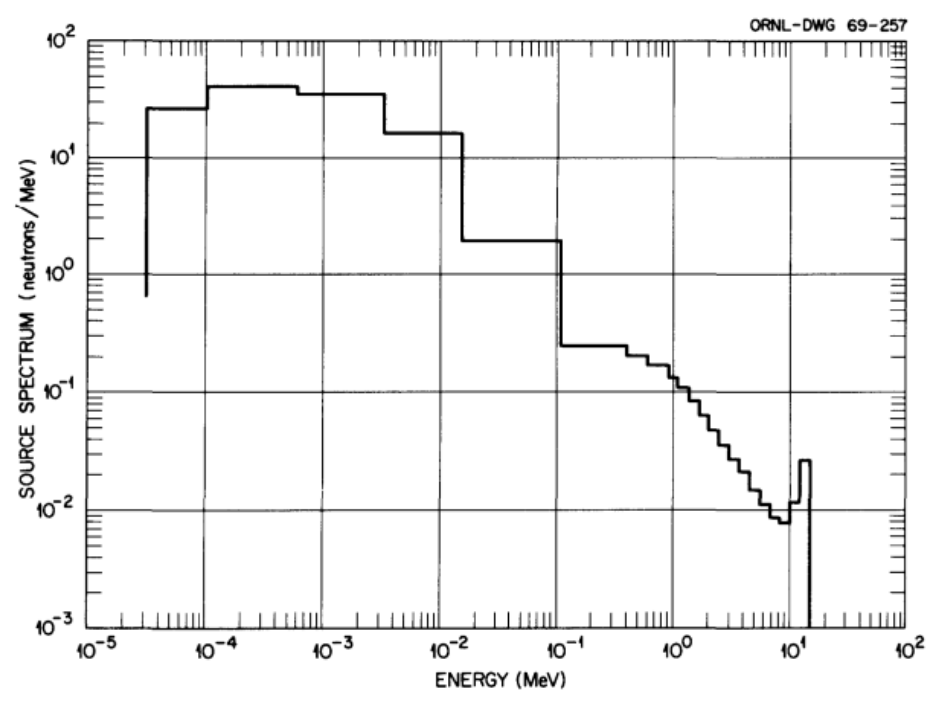




\section{Summary and Conclusion}

DIORAMA is a USNDS System Performance Modeling Tool with a focus on characterization of SNDD instrument performance.

It will replace existing fractured SNDD performance modeling tools with consolidated approach.

Much progress has been made in understanding the neutron transport problem and future work will include a transport functional form. 


\section{END}

\section{Thank you}

